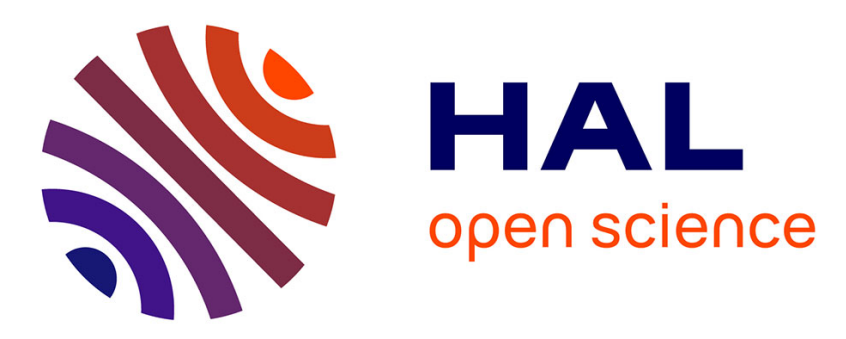

\title{
Plasticity coupled with thermo-electric fields: Thermodynamics framework and finite element method computations
}

Pablo Moreno-Navarro, Adnan Ibrahimbegovic, José L Pérez-Aparicio

\section{To cite this version:}

Pablo Moreno-Navarro, Adnan Ibrahimbegovic, José L Pérez-Aparicio. Plasticity coupled with thermo-electric fields: Thermodynamics framework and finite element method computations. Computer Methods in Applied Mechanics and Engineering, 2017, 315, pp.50-72. 10.1016/j.cma.2016.10.038 . hal-01993455

\section{HAL Id: hal-01993455 \\ https://hal.science/hal-01993455}

Submitted on 12 Feb 2019

HAL is a multi-disciplinary open access archive for the deposit and dissemination of scientific research documents, whether they are published or not. The documents may come from teaching and research institutions in France or abroad, or from public or private research centers.
L'archive ouverte pluridisciplinaire HAL, est destinée au dépôt et à la diffusion de documents scientifiques de niveau recherche, publiés ou non, émanant des établissements d'enseignement et de recherche français ou étrangers, des laboratoires publics ou privés. 


\title{
Plasticity coupled with thermo-electric fields: Thermodynamics framework and finite element method computations
}

\author{
Pablo Moreno-Navarro $^{\mathrm{a}, *}$, Adnan Ibrahimbegovic ${ }^{\mathrm{a}, *}$ José L. Pérez-Aparicio $^{\mathrm{b}}$ \\ ${ }^{a}$ Sorbonne Universités - Université de Technologie Compiègne, Laboratoire Roberval de Mécanique, Chaire de Mécanique, France \\ ${ }^{\mathrm{b}}$ Universitat Politècnica de València, Department of Continuum Mechanics \& Theory of Structures, Spain
}

Received 27 June 2016; received in revised form 20 October 2016; accepted 24 October 2016

Available online 1 November 2016

\section{Highlights}

- We present a complete formulation for thermo-electro-elastoplastic behavior that is developed in a consistent manner by using conservation principles along with the definition of a free-energy potential.

- FE implementation in the most general 3D framework, by using 8-node hexahedral finite element for constructing semidiscretization, along with the global phase of time discretization by the Newmark scheme.

- This development provides the sound basis for practical applications, such as the pulsed Peltier Cells.

\begin{abstract}
A consistent thermodynamic-based theoretical framework and three-dimensional finite element formulation is presented, capable of coupling elastic, thermal and electric fields. The complete set of governing equations is obtained from conservation principles for electric charge, energy and momentum. The second principle of thermodynamics is taken into account to introduce the irreversible phenomena, such as plastic dissipation or Joule heating. The constitutive relations are derived consistently from the Helmholtz free-energy potential for each corresponding dual variable in terms of the defined set of state variables. We consider the case of linear isotropic hardening model for plasticity, and provide the consistent form of the tangent thermo-electro-elastoplastic modulus through dual variable computations. The latter plays the crucial role in ensuring fast convergence properties of the finite element computations with the proposed coupled plasticity model. The implementation is carried out in a research version of the well-known computer code FEAP. Several numerical simulations are presented in order to illustrate the proposed model and formulation capabilities for providing an enhanced formulation of an important practical application in terms of Peltier cells.
\end{abstract}

(c) 2016 Elsevier B.V. All rights reserved.

Keywords: Thermo-electro-mechanics coupling; Plasticity; Thermodynamics; Finite element formulation

\footnotetext{
* Corresponding authors.

E-mail addresses: pablo.moreno-navarro@utc.fr (P. Moreno-Navarro), adnan.ibrahimbegovic@utc.fr (A. Ibrahimbegovic).
} 


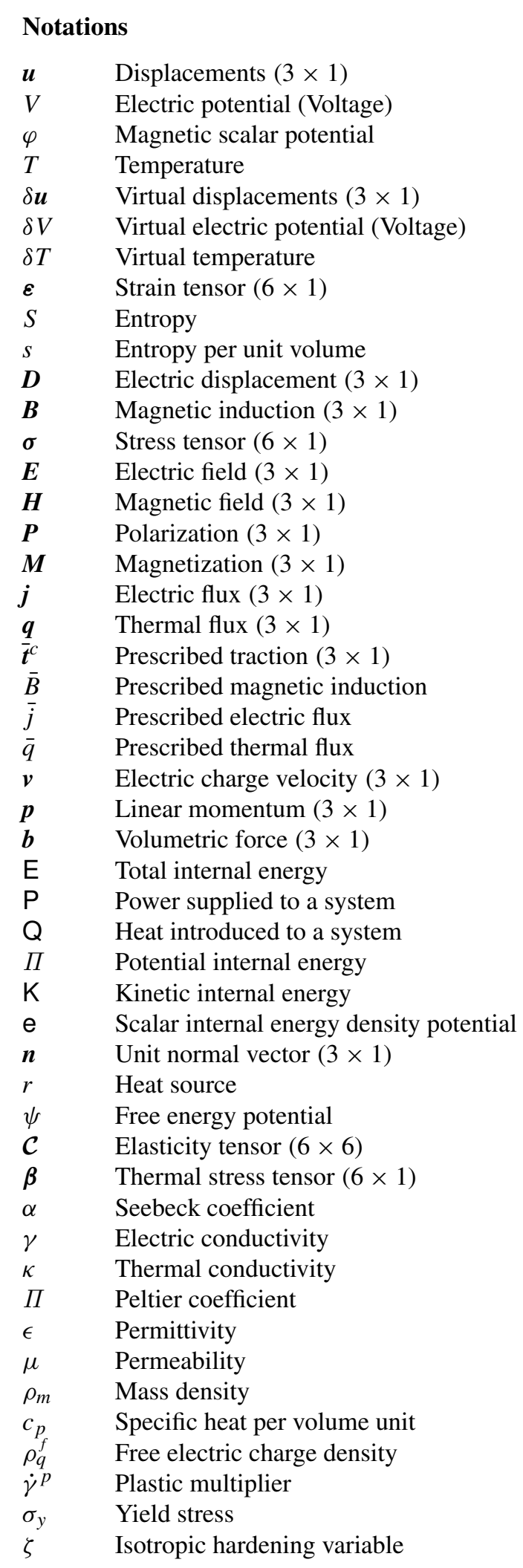




\begin{tabular}{|c|c|}
\hline$q$ & Stress-like conjugate to hardening variable \\
\hline$K$ & Isotropic hardening modulus \\
\hline $\mathscr{D}$ & Dissipation \\
\hline$\phi$ & Yield function \\
\hline$\omega$ & Coefficient of plastic influence \\
\hline$v$ & Derivative of $\phi$ with respect to $\sigma(6 \times 1)$ \\
\hline $\boldsymbol{I}$ & Identity tensor $(3 \times 3)$ \\
\hline 1 & Trace operator tensor $(3 \times 3)$ \\
\hline$\gamma, \beta$ & Newmark scheme parameters \\
\hline$\Delta t$ & Time step \\
\hline E & Young modulus \\
\hline$\alpha_{T}$ & Thermal expansion coefficient \\
\hline$\lambda, \mu$ & Lamé's parameters \\
\hline $\mathcal{S}$ & Effective tangent stiffness matrix (ndf $\times$ ndf) \\
\hline $\mathcal{L}$ & Linearized optimality equation matrix $12 \times 12$ \\
\hline$\overline{\mathcal{C}}, \hat{\mathcal{C}}$ & Thermo-electro-elastoplastic modulus matrix $10 \times 10$ \\
\hline $\mathcal{K}$ & Elastoplastic tangent stiffness matrix $(\mathrm{ndf} \times$ ndf) \\
\hline $\mathcal{D}$ & Damping matrix (ndf $\times$ ndf) \\
\hline $\mathcal{M}$ & Mass matrix $(\mathrm{ndf} \times \mathrm{ndf})$ \\
\hline $\mathcal{N}_{a}$ & Shape function \\
\hline $\mathcal{B}_{a}$ & Strain-displacement derivatives of shape function matrix $(3 \times 1)$ \\
\hline $\mathcal{B}_{a}^{s}$ & Symmetric part of the derivatives of shape function matrix $(6 \times 3)$ \\
\hline $\mathfrak{a}_{a}^{U}$ & Nodal displacement $(3 \times 1)$ \\
\hline $\mathfrak{a}_{a}^{V}$ & Nodal electric potential \\
\hline $\mathfrak{a}_{a}^{T}$ & Nodal temperature \\
\hline $\mathfrak{w}_{a}^{U}$ & Virtual nodal displacement $(3 \times 1)$ \\
\hline $\mathfrak{w}_{a}^{V}$ & Virtual nodal electric potential \\
\hline $\mathfrak{w}_{a}^{T}$ & Virtual nodal temperature \\
\hline $\mathcal{R}_{a}^{U}$ & Residual for displacement $(3 \times 1)$ \\
\hline $\mathcal{R}_{a}^{V}$ & Residual for electric potential \\
\hline $\mathcal{R}_{a}^{T}$ & Residual for temperature \\
\hline$\Omega$ & Domain \\
\hline$\Gamma$ & Domain boundary \\
\hline \multicolumn{2}{|c|}{ Sub-, Supra-indices } \\
\hline$e$ & Elastic \\
\hline$p$ & Plastic \\
\hline 0 & Reference or vacuum \\
\hline$C$ & Cauchy \\
\hline$M$ & Maxwell \\
\hline$L$ & Lorentz \\
\hline$J$ & Joule \\
\hline$c$ & Conduction \\
\hline $\mathrm{m}$ & Mechanic \\
\hline $\mathrm{e}$ & Electric \\
\hline h & Magnetic \\
\hline $\mathrm{t}$ & Thermal \\
\hline $\operatorname{tr}$ & Trial value \\
\hline$n$ & Time step \\
\hline
\end{tabular}




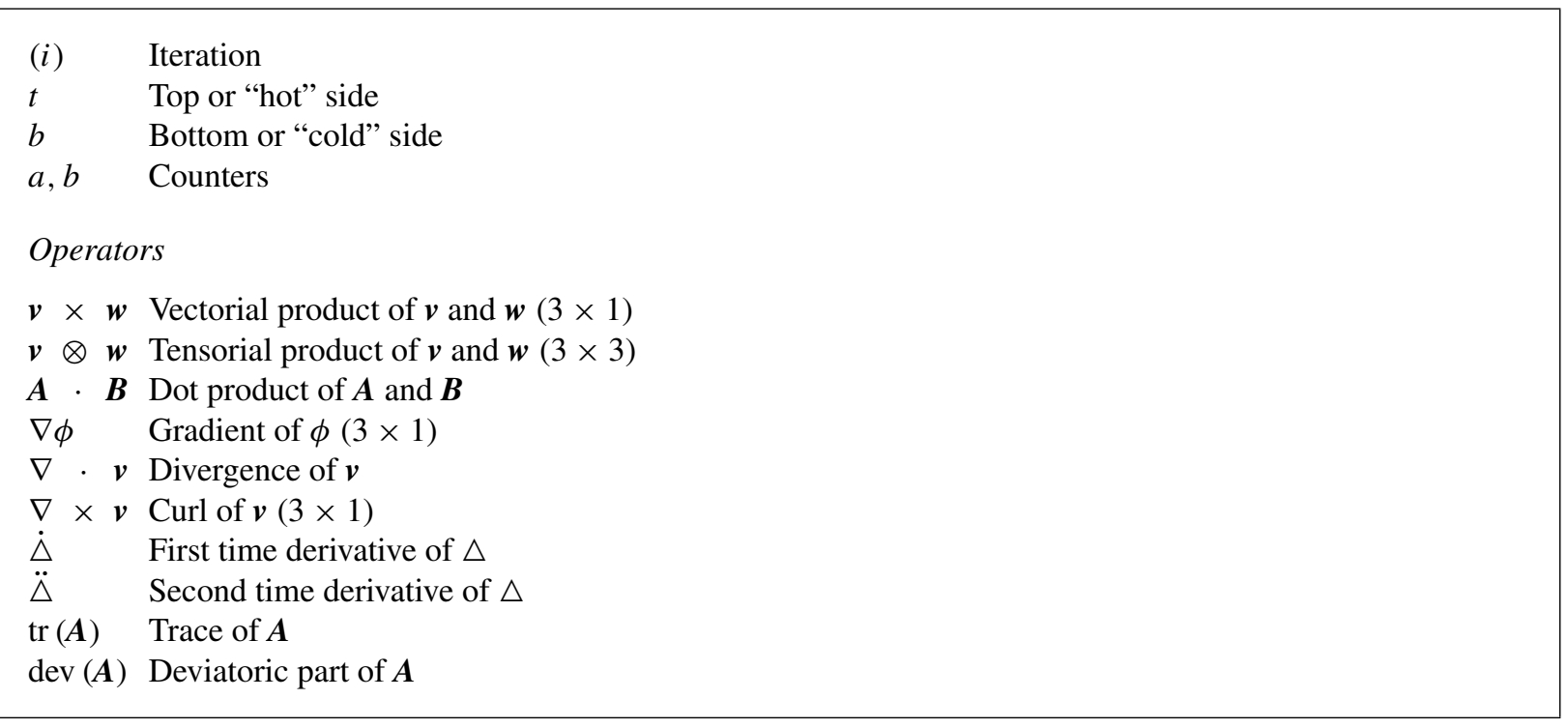

\section{Introduction}

This work seeks to provide a novel development of plasticity coupled with thermal and electric fields for semiconductor materials. The development is made fully consistent with thermodynamics point of view [1]. More precisely, the set of state variables is defined in terms of extensive variables [2] jointly defining the state of material. This is formally done in terms of the internal energy density, as the potential defined (at each point) indicating the current state of the material. The objective is twofold. First, this paper accounts for the complete set of state variables characterizing thermo-electro-mechanic coupling, which can all leave their imprint on a particular state of the given material. Second, this coupling is taken into inelastic domain defined in terms of plasticity theory that accounts for mechanical, thermal and electric fields in defining the corresponding plasticity criterion. The latter is set in terms of intensive variables, dual to the chosen state (extensive) variables. The corresponding evolution equations for plastic part of state variables are obtained from maximum dissipation principle. This theoretical formulation is accompanied by the discrete approximation, based upon 3D Finite Elements with thermo-electro-mechanic degrees of freedom, along with simultaneous solution procedure of the weak form for all governing equations.

The proposed approach provides a number of novelties with respect to previous developments. In particular, a vast majority of state-of-the-art developments remain limited to elastic response from early (e.g. [3] and [4]) or more recent works (e.g. [5]), and large portion will mostly focus upon electromagnetics (e.g. [6-9] and [10]). Several another consider the plasticity model for ferroelectrics (e.g. [11-14]), but without considering the numerical implementation, nor general multi-field coupling. In terms of completeness of presented developments for thermomechanics and electromagnetic coupling, the recent ones that come closest to this work are homogenization scheme in [15] and hypo-elastic approach in [16-18], but they remain limited to elastic behavior.

However, there is a fundamentally new point of view in this work, where the sound thermodynamics framework is brought to bear upon the general coupled problem of this kind. More precisely, our proposal starts from the internal or free-energy potential rather than directly postulating the constitutive equations. In other words, we provide the hyper-elasto-plastic response, rather than hypo-elastic response given in [17].

The outline of the paper is as follows. In Section 2, the sound thermodynamics-based theoretical formulation for coupled thermomechanical and electromagnetic problems is presented, along with the complete list of all pertinent principles. In Section 3, we further generalize such a development to the case of plasticity, by appealing to the principle of maximum dissipation to obtain the corresponding evolution equations with respect to the chosen plasticity criterion for generalized J2-plasticity accounting for thermal, electric and magnetic fields. The details of FE implementation, where the magnetic field has been removed for targeted applications, are presented in Section 4 for 3D case using the discrete approximation constructed with isoparametric finite elements of Peltier cells. The Newmark-type implicit time-integration scheme used for solving the FE discretization is presented in Section 5 as well as the iterative solution 
for the local variables and the computation of the consistent elastoplastic modulus. Several numerical simulations are presented in Section 6, and the concluding remarks are given in Section 7.

\section{Formulation}

\subsection{Kinematic equations}

Four fields are considered in formulating the thermomechanics and electromagnetics coupling: displacement $\boldsymbol{u}$, temperature $T$, electric potential $V$ and magnetic scalar potential $\varphi$. The state variables are obtained as the corresponding gradients of these fields. The set of resulting kinematic equations can be written as:

$$
\begin{aligned}
\boldsymbol{\varepsilon} & =\frac{1}{2}\left[\nabla \otimes \boldsymbol{u}+(\nabla \otimes \boldsymbol{u})^{\top}\right] \\
\boldsymbol{E} & =-\nabla V \\
\boldsymbol{H} & =-\nabla \varphi
\end{aligned}
$$

where $\boldsymbol{\varepsilon}$ is the strain tensor, $\boldsymbol{E}$ is the electric field, $\boldsymbol{H}$ is the magnetic field, while $\nabla=\left[\begin{array}{lll}\partial / \partial x & \partial / \partial y & \partial / \partial z\end{array}\right]^{\top}$ is a convenient notation for nabla operator of partial derivatives. With the hypothesis of small displacement gradient theory, we are limited here to strains defined in terms of the symmetric part of displacement gradient, hence the operator $\nabla^{s}$ can be used to define the strain tensor, which can also be written in Voigt notation as:

$$
\boldsymbol{\varepsilon}=\nabla^{s} \boldsymbol{u} ; \quad \nabla^{s}=\left[\begin{array}{ccc}
\frac{\partial}{\partial x} & 0 & 0 \\
0 & \frac{\partial}{\partial y} & 0 \\
0 & 0 & \frac{\partial}{\partial z} \\
\frac{\partial}{\partial y} & \frac{\partial}{\partial x} & 0 \\
0 & \frac{\partial}{\partial z} & \frac{\partial}{\partial y} \\
\frac{\partial}{\partial z} & 0 & \frac{\partial}{\partial x}
\end{array}\right] .
$$

\subsection{Conservation principles}

\subsubsection{Conservation of free electric charge}

Electric charge is the physical property of matter that causes it to experience a force when placed with an electromagnetic field. There are two types of electric charges related to their nature: free, which is associated with the movements of electrons in a conductor material, and bound, which is related to the orientation of dipoles in a dielectric.

The conservation of free charge can be written in agreement with the conservation equation:

$$
\dot{\rho}_{q}^{f}+\nabla \cdot\left(\rho_{q}^{f} v\right)=0
$$

where $\rho_{q}^{f}$ is the free electric charge density, $\boldsymbol{v}$ is the velocity of the charges. By identifying the electric flux as $\boldsymbol{j}=\rho_{q}^{f} \boldsymbol{v}$, the free-charge conservation equation can be rewritten as:

$$
\dot{\rho}_{q}^{f}+\nabla \cdot \boldsymbol{j}=0 .
$$

Furthermore, by considering the case with zero rate of electric charge density, $\dot{\rho}_{q}^{f}=0$, the first conservation equation reduces simply to:

$$
\nabla \cdot \boldsymbol{j}=0 .
$$


The last result is usually referred to as the Gauss Law for Electrostatics, stating that all the charges that come into the system will also have to leave. It is interesting to note that Maxwell's equations in differential form (e.g. [6]):

$$
\left\{\begin{array}{l}
\nabla \times \boldsymbol{E}=-\dot{\boldsymbol{B}} \\
\nabla \times \boldsymbol{H}=\boldsymbol{j}+\dot{\boldsymbol{D}} \\
\nabla \cdot \boldsymbol{D}=\rho_{q}^{f} \\
\nabla \cdot \boldsymbol{B}=0
\end{array}\right.
$$

where $\boldsymbol{D}$ is the electric displacement and $\boldsymbol{B}$ is the magnetic induction, can be used to confirm the result in (5). To that end, it is enough to apply the divergence operator to the second of Maxwell's equations and combine it with the time derivative of the third one.

We note in passing an analogous result for the magnetic field stating the non-existence of magnetic monopoles can be deducted from the fourth of (6) leading to Gauss Law for Magnetism.

\subsubsection{Conservation of momentum}

The classical approach to conservation of momentum (e.g. [1]) is now generalized accounting for the external electromagnetic Lorentz force. First, by enforcing the angular momentum conservation we obtain the symmetry of stress tensor (e.g. [1]). Second, by postulating linear momentum conservation principle for a sub-domain, and going to the limit of this sub-domain shrinking to a point, we obtain the local form of such a conservation equation:

$$
\underbrace{\rho_{m} \ddot{\boldsymbol{u}}}_{\dot{\boldsymbol{p}}_{\mathrm{m}}}=\boldsymbol{\sigma}^{C} \nabla+\boldsymbol{b}+\boldsymbol{b}_{L}
$$

where $\rho_{m}$ is the mass density, $\boldsymbol{\sigma}^{c}$ is the Cauchy stress tensor, $\boldsymbol{p}_{\mathrm{m}}$ is the mechanical linear momentum, $\boldsymbol{b}$ are the volume forces and $\boldsymbol{b}_{L}$ is the Lorentz force, which can be written as:

$$
\boldsymbol{b}_{L}:=\rho_{q}^{f} \boldsymbol{E}+\boldsymbol{j} \times \boldsymbol{B} .
$$

This force can be transformed using the second of Maxwell's equations (6) to eliminate $\boldsymbol{j}$. Similarly, in order to eliminate $\rho_{q}^{f}$, we can use the third equation in (6) along with the isotropic constitutive relations $\boldsymbol{D}=\epsilon \boldsymbol{E}$ and $\boldsymbol{B}=\mu \boldsymbol{H}$, with $\epsilon$ as the permittivity, $\mu$ as the permeability. An alternate way of expressing these relations (as suggested in [6]) by introducing the polarization $\boldsymbol{P}$ and the magnetization $\boldsymbol{M}$ :

$$
\begin{aligned}
& \boldsymbol{D}=\epsilon_{0} \boldsymbol{E}+\boldsymbol{P} \\
& \boldsymbol{B}=\mu_{0}(\boldsymbol{H}+\boldsymbol{M})
\end{aligned}
$$

where the subindex 0 refers to vacuum properties. This consideration is taken due to plasticity, introducing plastic electric displacement. Thus, (8) can be recast as follows:

$$
\rho_{q}^{f} \boldsymbol{E}+\boldsymbol{j} \times \boldsymbol{B}=\boldsymbol{E}(\nabla \cdot \boldsymbol{D})-\boldsymbol{B} \times(\nabla \times \boldsymbol{H})-\dot{\boldsymbol{D}} \times \boldsymbol{B} .
$$

The last term can be rewritten by exploiting the auxiliary result pertaining to the product rule:

$$
\frac{\partial}{\partial t}(\boldsymbol{D} \times \boldsymbol{B})=\dot{\boldsymbol{D}} \times \boldsymbol{B}+\boldsymbol{D} \times \dot{\boldsymbol{B}}
$$

and further using the first of Eqs. (6) in the last term, leading to:

$$
\rho_{q}^{f} \boldsymbol{E}+\boldsymbol{j} \times \boldsymbol{B}=\boldsymbol{E}(\nabla \cdot \boldsymbol{D})-\boldsymbol{B} \times(\nabla \times \boldsymbol{H})-\frac{\partial}{\partial t}(\boldsymbol{D} \times \boldsymbol{B})-\boldsymbol{D} \times(\nabla \times \boldsymbol{E}) .
$$

Adding to the right side of the previous expression the null term $\boldsymbol{H}(\nabla \cdot \boldsymbol{B})$ and reordering, we finally obtain:

$$
\rho_{q}^{f} \boldsymbol{E}+\boldsymbol{j} \times \boldsymbol{B}=-\frac{\partial}{\partial t} \underbrace{(\boldsymbol{D} \times \boldsymbol{B})}_{\mathrm{I}}+\underbrace{\boldsymbol{E}(\nabla \cdot \boldsymbol{D})-\boldsymbol{D} \times(\nabla \times \boldsymbol{E})+\boldsymbol{H}(\nabla \cdot \boldsymbol{B})-\boldsymbol{B} \times(\nabla \times \boldsymbol{H})}_{\mathrm{II}} .
$$


Table 1

List of the state variables and their corresponding dual variables for coupled thermoelasticity and electromagnetism.

\begin{tabular}{lllll}
\hline Fields & Mechanic & Thermal & Electric & Magnetic \\
\hline State var. & $\boldsymbol{\varepsilon}$ & $s$ & $\boldsymbol{D}$ & $\boldsymbol{B}$ \\
Dual var. & $\boldsymbol{\sigma}$ & $T$ & $\boldsymbol{E}$ & $\boldsymbol{H}$ \\
\hline
\end{tabular}

The term I is the electromagnetic momentum $p_{\text {eh }}$ and the term II can be expressed in the form $\sigma^{M} \nabla$, where $\sigma^{M}$ is the Maxwell stress tensor:

$$
\boldsymbol{\sigma}^{M}=\epsilon \boldsymbol{E} \otimes \boldsymbol{E}+\mu \boldsymbol{H} \otimes \boldsymbol{H}-\frac{\boldsymbol{I}}{2}(\epsilon \boldsymbol{E} \cdot \boldsymbol{E}+\mu \boldsymbol{H} \cdot \boldsymbol{H})
$$

with $\boldsymbol{I}$ denoting unit second order tensor. In (13) above, the electromagnetic momentum can be neglected due to the different order of magnitude between the time constants of the mechanic and the electromagnetic fields; we thus obtain an alternative form of momentum balance equation in (7), which can be written as:

$$
\rho_{m} \ddot{\boldsymbol{u}}=\boldsymbol{\sigma} \nabla+\boldsymbol{b}
$$

where $\sigma=\sigma^{C}+\sigma^{M}$ is the total stress tensor, regrouping the Cauchy and the Maxwell terms.

\subsubsection{Conservation of energy}

The global form of the first principle of thermodynamics can be stated in domain $\Omega$ :

$$
\frac{\partial}{\partial t} E=P_{m}+P_{e h}+Q
$$

where $E$ is the total energy, $P_{m}$ is the mechanical power, $P_{\text {eh }}$ is the electromagnetic power and $Q$ is the total heat supplied to the system. The total energy can further be split into the potential $\Pi$ and kinetic $\mathrm{K}$ energy:

$$
\mathrm{E}=\Pi+\mathrm{K}=\int_{\Omega} \mathrm{e}(\boldsymbol{\varepsilon}, s, \boldsymbol{D}, \boldsymbol{B}) \mathrm{d} \Omega+\frac{1}{2} \int_{\Omega} \rho_{m} \dot{\boldsymbol{u}} \cdot \dot{\boldsymbol{u}} \mathrm{d} \Omega
$$

where $e$ is the scalar potential of the internal energy density that depends on the state variables. The list of state variables in the absence of plasticity is as defined in Table 1, along with their corresponding dual variables (with $s$ is the entropy per unit volume).

The mechanical power source inserted into the material within particular domain $\Omega$ can be written:

$$
\mathrm{P}_{\mathrm{m}}:=\int_{\Omega} \boldsymbol{b} \cdot \dot{\boldsymbol{u}} \mathrm{d} \Omega+\int_{\Gamma} \boldsymbol{t}^{n} \cdot \dot{\boldsymbol{u}} \mathrm{d} \Gamma=\int_{\Omega} \boldsymbol{b} \cdot \dot{\boldsymbol{u}} \mathrm{d} \Omega+\int_{\Omega} \nabla \cdot(\sigma \dot{\boldsymbol{u}}) \mathrm{d} \Omega
$$

where the power source from the boundary traction vector, defined with respect to unit normal vector $\boldsymbol{n}$ by the Cauchy principle $\boldsymbol{t}^{n}=\sigma \boldsymbol{n}$, has been transformed into corresponding volume integral by use of the divergence theorem. Similarly, the boundary term for electromagnetic power source (see [6]) is transformed into corresponding volume integral defined as:

$$
\mathrm{P}_{\mathrm{eh}}:=-\int_{\Gamma}(\boldsymbol{E} \times \boldsymbol{H}) \cdot \boldsymbol{n} \mathrm{d} \Gamma=-\int_{\Omega} \nabla \cdot(\boldsymbol{E} \times \boldsymbol{H}) \mathrm{d} \Omega .
$$

We note in passing that the negative sign is in agreement with the above expression representation of total power exiting the volume $\Omega$ bounded by the surface $\Gamma$. Similar interpretation for negative sign holds for the heat power source that stems from the outgoing heat flux $\boldsymbol{q}$, along with the heat source $r$, which can be stated as:

$$
\mathrm{Q}:=\int_{\Omega} r \mathrm{~d} \Omega-\int_{\Gamma} \boldsymbol{q} \cdot \boldsymbol{n} \mathrm{d} \Gamma=\int_{\Omega} r \mathrm{~d} \Omega-\int_{\Omega} \nabla \cdot \boldsymbol{q} \mathrm{d} \Omega
$$


In the limit case of domain $\Omega$ shrinking to a point, we obtain from (16) the local form of the first principle that can be written as:

$$
\dot{\mathrm{e}}(\boldsymbol{\varepsilon}, s, \boldsymbol{D}, \boldsymbol{B})+\rho_{m} \ddot{\boldsymbol{u}} \cdot \dot{\boldsymbol{u}}=\boldsymbol{b} \cdot \dot{\boldsymbol{u}}+\nabla \cdot(\sigma \dot{\boldsymbol{u}})+r-\nabla \cdot \boldsymbol{q}-\nabla \cdot(\boldsymbol{E} \times \boldsymbol{H}) .
$$

By using furthermore the kinematic equations in (1) and the equation of motion in (15), along with the following identity $\nabla \cdot(\sigma \dot{\boldsymbol{u}})=(\sigma \nabla) \cdot \dot{\boldsymbol{u}}+\sigma \cdot(\nabla \otimes \dot{\boldsymbol{u}})$, we can obtain the reduced form of energy conservation principle:

$$
\dot{\mathrm{e}}(\boldsymbol{\varepsilon}, s, \boldsymbol{D}, \boldsymbol{B})=\boldsymbol{\sigma} \cdot \dot{\boldsymbol{\varepsilon}}+r-\nabla \cdot \boldsymbol{q}-\nabla \cdot(\boldsymbol{E} \times \boldsymbol{H}) .
$$

The final ingredients pertain to provide definition of electric and heat fluxes through the generalized form of Ohm's and Fourier's laws, as suggested in [10] and [17]:

$$
\begin{gathered}
\boldsymbol{j}=\gamma \boldsymbol{E}-\gamma \alpha \nabla T ; \\
\boldsymbol{q}=-\kappa \nabla T+\Pi \boldsymbol{j}
\end{gathered}
$$

where $\gamma$ is the electric conductivity, $\alpha$ is the Seebeck coefficient, $\kappa$ is the thermal conductivity and $\Pi=T \alpha$ is the Peltier coefficient.

\section{Plasticity under thermo-electro-mechanics coupling}

We seek to develop here the general form of associative plasticity for a coupled problem of this kind. Such a development will first require the use of the second principle of thermodynamics in order to define the corresponding dissipation. For a particular domain $\Omega$, the second principle imposes that the rate of increase of entropy $S=\int_{\Omega} s \mathrm{~d} \Omega$ should never be smaller than the amount of heat divided by the absolute temperature, which we can write as:

$$
\dot{S} \geq \frac{\mathrm{Q}}{T}
$$

In the limit case of shrinking this domain to a point, we can obtain the local form of the second principle. In the simplest case of rigid conductor (corresponding to the neglection of all other fields but temperature) the second principle provides the proper definition of dissipation by heat conduction [1], which is always positive as long as the Fourier law applies:

$$
-\underbrace{\frac{1}{T} \boldsymbol{q} \cdot \nabla T}_{\mathscr{D} C}:=T \dot{s}-(r-\nabla \cdot \boldsymbol{q}) \geq 0 .
$$

Here, we are targeting much more general case of loading program with corresponding contributions from all fields. The second principle, combined with the result of the first principle in (22), can now be used to define the local dissipation that always remain non-negative:

$$
\mathscr{D}:=T \dot{s}-\dot{\mathrm{e}}(\boldsymbol{\varepsilon}, s, \boldsymbol{D}, \boldsymbol{B})+\boldsymbol{\sigma} \cdot \dot{\boldsymbol{\varepsilon}}-\nabla \cdot(\boldsymbol{E} \times \boldsymbol{H}) \geq 0
$$

where we dropped the dissipation by conduction. Furthermore, by scalar multiplying the two first Maxwell's equations in (6) with $\boldsymbol{H}$ and $\boldsymbol{E}$ respectively, and by exploiting the following identity:

$$
\nabla \cdot(\boldsymbol{E} \times \boldsymbol{H})=\boldsymbol{H} \cdot(\nabla \times \boldsymbol{E})-\boldsymbol{E} \cdot(\nabla \times \boldsymbol{H})
$$

the local dissipation can be recast in an equivalent format:

$$
\mathscr{D}=T \dot{s}-\dot{\mathrm{e}}+\boldsymbol{\sigma} \cdot \dot{\boldsymbol{\varepsilon}}+\boldsymbol{j} \cdot \boldsymbol{E}+\boldsymbol{E} \cdot \dot{\boldsymbol{D}}+\boldsymbol{H} \cdot \dot{\boldsymbol{B}} \geq 0 .
$$

We next turn to the case when plasticity is activated. In the framework of linear kinematics defined in (1), it is generally accepted to use the additive decomposition of the state variables into elastic and plastic parts:

$$
\begin{aligned}
& \boldsymbol{\varepsilon}=\boldsymbol{\varepsilon}^{e}+\boldsymbol{\varepsilon}^{p} \\
& s=s^{e}+s^{p} \\
& \boldsymbol{D}=\boldsymbol{D}^{e}+\boldsymbol{D}^{p} \\
& \boldsymbol{B}=\boldsymbol{B}^{e}+\boldsymbol{B}^{p}
\end{aligned}
$$


The internal energy potential is now defined in terms of the elastic part of the state variables $\boldsymbol{\varepsilon}^{e}, s^{e}, \boldsymbol{D}^{e}, \boldsymbol{B}^{e}$. For describing eventual hardening phenomena, which makes the model more predictive than perfect plasticity [1], we also need to add a new state variable that can monitor the progress of plasticity threshold, here chosen in terms of isotropic hardening variable $\zeta$. Furthermore, we introduce the free energy potential $\psi$ by means of the Legendre transformation (e.g. [1]), which allows to exchange the roles between the state variables and their duals, $s^{e}, \boldsymbol{D}^{e}, \boldsymbol{B}^{e}$ versus $T, \boldsymbol{E}, \boldsymbol{H}$ :

$$
\psi\left(\boldsymbol{\varepsilon}^{e}, \zeta, T, \boldsymbol{E}, \boldsymbol{H}\right)=\mathrm{e}\left(\boldsymbol{\varepsilon}^{e}, \zeta, s^{e}, \boldsymbol{D}^{e}, \boldsymbol{B}^{e}\right)-T s^{e}-\boldsymbol{E} \cdot \boldsymbol{D}^{e}-\boldsymbol{H} \cdot \boldsymbol{B}^{e} .
$$

The time derivative of the last expression leads to:

$$
\begin{aligned}
\dot{\mathrm{e}}= & \frac{\partial \psi}{\partial \boldsymbol{\varepsilon}^{e}} \cdot \dot{\boldsymbol{\varepsilon}}^{e}+\underbrace{\frac{\partial \psi}{\partial \zeta}}_{-q} \dot{\zeta}+\frac{\partial \psi}{\partial T} \dot{T}+\frac{\partial \psi}{\partial \boldsymbol{E}} \cdot \dot{\boldsymbol{E}}+\frac{\partial \psi}{\partial \boldsymbol{H}} \cdot \dot{\boldsymbol{H}}+\dot{T} s^{e}+T \dot{s}^{e}+\boldsymbol{E} \cdot \dot{\boldsymbol{D}}^{e} \\
& +\dot{\boldsymbol{E}} \cdot \boldsymbol{D}^{e}+\boldsymbol{H} \cdot \dot{\boldsymbol{B}}^{e}+\dot{\boldsymbol{H}} \cdot \boldsymbol{B}^{e}
\end{aligned}
$$

where $q$ is stress-like variable thermodynamically conjugate to $\zeta$, which controls the evolution of plasticity threshold. With this result on hand, the dissipation in (28) can be expressed as:

$$
\begin{aligned}
0 \leq \mathscr{D}= & \left(\boldsymbol{\sigma}-\frac{\partial \psi}{\partial \boldsymbol{\varepsilon}^{e}}\right) \cdot \dot{\boldsymbol{\varepsilon}}^{e}-\left(s^{e}+\frac{\partial \psi}{\partial T}\right) \dot{T}-\left(\boldsymbol{D}^{e}+\frac{\partial \psi}{\partial \boldsymbol{E}}\right) \cdot \dot{\boldsymbol{E}}-\left(\boldsymbol{B}^{e}+\frac{\partial \psi}{\partial \boldsymbol{H}}\right) \cdot \dot{\boldsymbol{H}} \\
& +q \dot{\zeta}+\boldsymbol{\sigma} \cdot \dot{\boldsymbol{\varepsilon}}^{p}+T \dot{s}^{p}+\boldsymbol{E} \cdot \dot{\boldsymbol{D}}^{p}+\boldsymbol{H} \cdot \dot{\boldsymbol{B}}^{p}+\boldsymbol{j} \cdot \boldsymbol{E} .
\end{aligned}
$$

The last term is often referred to as Joule's dissipation, which can be written as:

$$
\mathscr{D}^{J}=\boldsymbol{j} \cdot \boldsymbol{E} \text {. }
$$

By considering the elastic process, where (possibly non-zero values of) internal variables remain frozen enforcing that $\dot{\boldsymbol{\varepsilon}}^{p}=\mathbf{0}, \dot{\zeta}=0, \dot{\boldsymbol{s}}^{p}=0, \dot{\boldsymbol{D}}^{p}=\mathbf{0}, \dot{\boldsymbol{B}}^{p}=\mathbf{0}$, and putting aside the Joule's dissipation, we can conclude that the local plastic dissipation remains equal to zero. The dissipation inequality will become an equality providing the set of constitutive equations to be defined in agreement with the chosen free energy potential. Here, we choose a quadratic form of that potential, which can be written as follows:

$$
\begin{array}{rlrl} 
& \psi\left(\boldsymbol{\varepsilon}^{e}, \zeta, T, \boldsymbol{E}, \boldsymbol{H}\right)=\psi_{\mathrm{m}}+\psi_{\mathrm{p}}+\psi_{\mathrm{t}}+\psi_{\mathrm{e}}+\psi_{\mathrm{h}}+\psi_{\mathrm{mt}}+\psi_{\mathrm{me}}+\psi_{\mathrm{mh}} ; \\
\psi_{\mathrm{m}}= & \frac{1}{2} \boldsymbol{\varepsilon}^{e} \cdot \mathcal{C} \boldsymbol{\varepsilon}^{e} ; & \psi_{\mathrm{h}}=-\frac{1}{2} \boldsymbol{H} \cdot \mu \boldsymbol{H} ; \\
\psi_{\mathrm{p}}=\frac{1}{2} \zeta K \zeta ; & \psi_{\mathrm{mt}}=-\boldsymbol{\beta} \cdot\left(T-T_{0}\right) \boldsymbol{\varepsilon}^{e} ; \\
\psi_{\mathrm{t}}=\rho_{m} c_{p}\left[\left(T-T_{0}\right)-T \ln \frac{T}{T_{0}}\right] ; & \psi_{\mathrm{me}}=\epsilon\left[(\boldsymbol{E} \otimes \boldsymbol{E}) \cdot \boldsymbol{\varepsilon}^{e}-\frac{1}{2}(\boldsymbol{E} \cdot \boldsymbol{E}) \operatorname{tr}\left(\boldsymbol{\varepsilon}^{e}\right)\right] ; \\
\psi_{\mathrm{e}}=-\frac{1}{2} \boldsymbol{E} \cdot \epsilon \boldsymbol{E} ; & \psi_{\mathrm{mh}}=\mu\left[(\boldsymbol{H} \otimes \boldsymbol{H}) \cdot \boldsymbol{\varepsilon}^{e}-\frac{1}{2}(\boldsymbol{H} \cdot \boldsymbol{H}) \operatorname{tr}\left(\boldsymbol{\varepsilon}^{e}\right)\right]
\end{array}
$$

where $\mathcal{C}$ is the elasticity tensor, $K$ is the isotropic hardening modulus, $c_{p}$ is the specific heat and $\boldsymbol{\beta}=\mathcal{C} \alpha_{T} \boldsymbol{I}$ is the thermal stress tensor with $\alpha_{T}$ as the expansion coefficient. The constitutive equations can then be obtained from derivatives of such a potential, and written in tensor notation:

$$
\left\{\begin{aligned}
\boldsymbol{\sigma} & :=\frac{\partial \psi}{\partial \boldsymbol{\varepsilon}^{e}}=\mathcal{C}\left(\boldsymbol{\varepsilon}-\boldsymbol{\varepsilon}^{p}\right)-\boldsymbol{\beta}\left(T-T_{0}\right)+\epsilon \boldsymbol{E} \otimes \boldsymbol{E}+\mu \boldsymbol{H} \otimes \boldsymbol{H}-\frac{\boldsymbol{I}}{2}(\epsilon \boldsymbol{E} \cdot \boldsymbol{E}+\mu \boldsymbol{H} \cdot \boldsymbol{H}) \\
\boldsymbol{D}^{e} & :=-\frac{\partial \psi}{\partial \boldsymbol{E}}=\epsilon\left\{\left[1+\operatorname{tr}\left(\boldsymbol{\varepsilon}^{e}\right)\right] \boldsymbol{E}-\boldsymbol{\varepsilon}^{e} \boldsymbol{E}\right\} \\
\boldsymbol{B}^{e} & :=-\frac{\partial \psi}{\partial \boldsymbol{H}}=\mu\left\{\left[1+\operatorname{tr}\left(\boldsymbol{\varepsilon}^{e}\right)\right] \boldsymbol{H}-\boldsymbol{\varepsilon}^{e} \boldsymbol{H}\right\} \\
q & :=-\frac{\partial \psi}{\partial \zeta}=-K \zeta \\
s^{e} & :=-\frac{\partial \psi}{\partial T}=\rho_{m} c_{p} \ln \left(\frac{T}{T_{0}}\right)+\boldsymbol{\beta} \cdot\left(\boldsymbol{\varepsilon}-\boldsymbol{\varepsilon}^{p}\right) .
\end{aligned}\right.
$$


By assuming that such constitutive equations also remain valid in an inelastic process, the dissipation can now be defined as the sum of plastic and Joule terms. Furthermore, the plastic dissipation can be split into mechanic, thermal and electromagnetic terms defined explicitly as:

$$
\begin{aligned}
& \mathscr{D}=\mathscr{D}^{p}+\mathscr{D}^{J} ; \\
& \mathscr{D}^{p}=\underbrace{q \dot{\zeta}+\boldsymbol{\sigma} \cdot \dot{\boldsymbol{\varepsilon}}^{p}}_{\mathscr{D}_{\mathrm{m}}^{p}}+\underbrace{T \dot{s}^{p}}_{\mathscr{D}_{\mathrm{t}}^{p}}+\underbrace{\boldsymbol{E} \cdot \dot{\boldsymbol{D}}^{p}+\boldsymbol{H} \cdot \dot{\boldsymbol{B}}^{p}}_{\mathscr{D}_{\mathrm{eh}}^{p}} .
\end{aligned}
$$

With these results on hand, we can rewrite (28) in terms of generalizing the heat equation for inelastic case which can be written as:

$$
T \dot{s}=r-\nabla \cdot \boldsymbol{q}+\mathscr{D} \quad \Rightarrow \quad T \dot{s}^{e}=r-\nabla \cdot \boldsymbol{q}+\mathscr{D}_{\mathrm{m}}^{p}+\mathscr{D}_{\mathrm{eh}}^{p}+\mathscr{D}^{J}
$$

where the results (29) and (36) are used. Furthermore, by using the constitutive equation for $s^{e}$ in (35), we can write explicitly:

$$
T \dot{s}^{e}:=\rho_{m} c_{p} \dot{T}+T \boldsymbol{\beta} \cdot\left(\dot{\boldsymbol{\varepsilon}}-\dot{\boldsymbol{\varepsilon}}^{p}\right) .
$$

By introducing (38) into (37) and using (35), the generalized heat equation can finally be restated as:

$$
\rho_{m} c_{p} \dot{T}=r-\nabla \cdot \boldsymbol{q}-T \boldsymbol{\beta} \cdot\left(\dot{\boldsymbol{\varepsilon}}-\dot{\boldsymbol{\varepsilon}}^{p}\right)+\mathscr{D}^{J}+\mathscr{D}_{\mathrm{m}}^{p}+\mathscr{D}_{\mathrm{eh}}^{p} .
$$

In summary, the strong form of the equations to be solved regroups the results written in (5), (6), (15) and (39), here restated in tensor notation:

$$
\left\{\begin{aligned}
\rho_{m} \ddot{\boldsymbol{u}} & =\nabla \cdot \boldsymbol{\sigma}+\boldsymbol{b} \\
\nabla \cdot \boldsymbol{j} & =0 \\
\nabla \cdot \boldsymbol{B} & =0 \\
\rho_{m} c_{p} \dot{T} & =r-\nabla \cdot \boldsymbol{q}-T \boldsymbol{\beta} \cdot\left(\dot{\boldsymbol{\varepsilon}}-\dot{\boldsymbol{\varepsilon}}^{p}\right)+\mathscr{D}^{J}+\mathscr{D}_{\mathrm{m}}^{p}+\mathscr{D}_{\mathrm{eh}}^{p} .
\end{aligned}\right.
$$

\subsection{Evolution equations for internal variables}

We will further consider the case where magnetic field does not have influence in the applications studied in this paper, so that it can be dropped from subsequent developments considering it remains equal to zero. This includes a number of practical applications, such as Peltier cells, where we need to account for plasticity phenomena in metallic materials. We here use generalized form of von Mises criterion that builds upon the proposal in [12] for perfect plasticity, which allows to determine if the material is in elastic or plastic regime based upon its stress state and electric field values. The first generalization of such criterion concerns the possibility to include the thermal field, leading to:

$$
\phi(\boldsymbol{\sigma}, q, T, \boldsymbol{E}):=\frac{3}{2} \frac{\|\operatorname{dev}(\boldsymbol{\sigma})\|^{2}}{\sigma_{y}^{2}(T)}+\frac{\|\boldsymbol{E}\|^{2}}{E_{0}^{2}}-1=0
$$

where $\operatorname{dev}(\boldsymbol{A})=\boldsymbol{A}-\frac{1}{3}[\operatorname{tr}(\boldsymbol{A})]$ is the deviatoric part of the tensor $\boldsymbol{A},\|\boldsymbol{A}\|=\sqrt{\operatorname{tr}\left(\boldsymbol{A}^{\top} \boldsymbol{A}\right)}$ is the Euclidean norm of $\boldsymbol{A}$, $\sigma_{y}(T)$ is the temperature dependent yield stress and $E_{0}$ a reference value.

The same criterion can be recast in a more standard format (e.g. [1]) by making the yield stress dependent upon thermal and electric field variations, which also allows to account for potential hardening effect in terms of isotropic hardening. Such a generalized von Mises criterion can be written as:

$$
\phi(\boldsymbol{\sigma}, q, T, \boldsymbol{E})=\|\operatorname{dev}(\boldsymbol{\sigma})\|-\sqrt{\frac{2}{3}}\left[\sigma_{y}(T, \boldsymbol{E})-q(T, \boldsymbol{E})\right]=0 ; q=-K(T, \boldsymbol{E}) \zeta
$$

where the yield stress and the isotropic hardening modulus are now assumed dependent upon thermal and electric fields, according to the following dependency:

$$
\begin{aligned}
\sigma_{y}(T, \boldsymbol{E}) & =\sigma_{y_{0}}\left[1-\omega_{\mathrm{t}}\left(T-T_{0}\right)\right]\left[1-\omega_{\mathrm{e}}\left(\|\boldsymbol{E}\|-E_{0}\right)\right] \\
K(T, \boldsymbol{E}) & =K_{0}\left[1-\omega_{\mathrm{t}}\left(T-T_{0}\right)\right]\left[1-\omega_{\mathrm{e}}\left(\|\boldsymbol{E}\|-E_{0}\right)\right]
\end{aligned}
$$


where $\omega_{\mathrm{t}}$ and $\omega_{\mathrm{e}}$ are some coefficients to define the influence of the temperature and the electric fields respectively in $\sigma_{y}$ and $K$.

By using the principle of maximum dissipation [1], we will pick among all plastically admissible values of dual variables (which satisfy $\phi(\sigma, q, T, \boldsymbol{E})=0$ ) the one which will maximize the plastic dissipation. We can further recast such a constrained optimization problem in terms of min-max problem of unconstrained minimization where the plastic admissibility constraint is enforced by using the method of Lagrange multipliers leading to plastic Lagrangian:

$$
\max _{\phi(\boldsymbol{\sigma}, q, T, \boldsymbol{E})=0} \mathscr{D}^{p}(\boldsymbol{\sigma}, q, T, \boldsymbol{E}) \Leftrightarrow \min _{\forall(\boldsymbol{\sigma}, q, T, \boldsymbol{E})} \max _{\dot{\gamma}^{p}>0} \mathscr{L}^{p}\left(\boldsymbol{\sigma}, q, T, \boldsymbol{E}, \dot{\gamma}^{p}\right)
$$

where the Lagrange multiplier is equal to the plastic multiplier $\dot{\gamma}^{p}$. The plastic Lagrangian will incorporate the yield criterion constraint $\phi=0$, which can be written as:

$$
\mathscr{L}^{p}\left(\sigma, q, T, \boldsymbol{E}, \dot{\gamma}^{p}\right):=-\mathscr{D}^{p}(\boldsymbol{\sigma}, q, T, \boldsymbol{E})+\dot{\gamma}^{p} \phi(\boldsymbol{\sigma}, q, T, \boldsymbol{E}) .
$$

The solution to such constrained minimization problem can be obtained by using the Kuhn-Tucker optimality conditions, which allows to write:

$$
\begin{aligned}
\mathbf{0} & :=\frac{\partial \mathscr{L}^{p}}{\partial \boldsymbol{\sigma}}=\dot{\gamma}^{p} \frac{\partial \phi}{\partial \boldsymbol{\sigma}}-\dot{\boldsymbol{\varepsilon}}^{p} ; \\
0 & :=\frac{\partial \mathscr{L}^{p}}{\partial q}=\dot{\gamma}^{p} \frac{\partial \phi}{\partial q}-\dot{\zeta} ; \\
0 & :=\frac{\partial \mathscr{L}^{p}}{\partial T}=\dot{\gamma}^{p} \frac{\partial \phi}{\partial T}-\dot{s}^{p} ; \\
\mathbf{0} & :=\frac{\partial \mathscr{L}^{p}}{\partial \boldsymbol{E}}=\dot{\gamma}^{p} \frac{\partial \phi}{\partial \boldsymbol{E}}-\dot{\boldsymbol{D}}^{p} ; \\
\dot{\gamma}^{p} & \geq 0 ; \quad \phi \leq 0 ; \quad \dot{\gamma}^{p} \phi=0 .
\end{aligned}
$$

Using the first two previous equations, $\mathscr{D}_{\mathrm{m}}^{p}$ can further be simplified:

$$
\mathscr{D}_{\mathrm{m}}^{p}=\dot{\gamma}^{p} \overbrace{[\underbrace{\boldsymbol{\sigma} \cdot \boldsymbol{v}}_{\|\operatorname{dev}(\boldsymbol{\sigma})\|}-\sqrt{\frac{2}{3}}\left(\sigma_{y}-q\right)]}^{\phi=0}+\dot{\gamma}^{p} \sqrt{\frac{2}{3}} \sigma_{y}=\dot{\gamma}^{p} \sqrt{\frac{2}{3}} \sigma_{y}
$$

where $\boldsymbol{v}:=\partial \phi / \partial \boldsymbol{\sigma}=\operatorname{dev}(\boldsymbol{\sigma}) /\|\operatorname{dev}(\boldsymbol{\sigma})\|$.

\section{Finite element implementation}

In this section we present the details of the discrete approximation constructed by the finite element method, as a particular case of the Galerkin method. The starting point is provided by the weak form of the conservation equations in (40), which can be stated in tensor notation as follows:

$$
\left\{\begin{array}{l}
-\int_{\Omega} \nabla^{s} \delta \boldsymbol{u} \cdot \boldsymbol{\sigma}+\delta \boldsymbol{u} \cdot\left(\rho_{m} \ddot{\boldsymbol{u}}-\boldsymbol{b}\right) \mathrm{d} \Omega+\int_{\Gamma} \delta \boldsymbol{u} \cdot \overline{\boldsymbol{t}}^{c} \mathrm{~d} \Gamma=0 \\
\int_{\Omega} \nabla \delta V \cdot \boldsymbol{j} \mathrm{d} \Omega-\int_{\Gamma} \delta V \bar{j} \mathrm{~d} \Gamma=0 \\
\int_{\Omega} \nabla \delta T \cdot \boldsymbol{q}-\delta T\left[\rho_{m} c_{p} \dot{T}-r+T \boldsymbol{\beta} \cdot\left(\dot{\boldsymbol{\varepsilon}}-\dot{\boldsymbol{\varepsilon}}^{p}\right)-\boldsymbol{j} \cdot \boldsymbol{E}-\dot{\gamma}^{p} \sqrt{\frac{2}{3}} \sigma_{y}-\boldsymbol{E} \cdot \dot{\boldsymbol{D}}^{p}-\boldsymbol{H} \cdot \dot{\boldsymbol{B}}^{p}\right] \mathrm{d} \Omega \\
\quad-\int_{\Gamma} \delta T \bar{q} \mathrm{~d} \Gamma=0
\end{array}\right.
$$

where $\delta \bullet$ denotes a virtual field or variation. It can be noticed that the conservation equation for the magnetic field has been dropped as already stated above. We can readily obtain the discrete approximations for all the fields, along with 
their space and time derivatives by appealing to separation of variables:

$$
\begin{aligned}
& \boldsymbol{u} \approx \mathcal{N}_{b} \mathfrak{a}_{b}^{U} ; \quad V \approx \mathcal{N}_{b} \mathfrak{a}_{b}^{V} ; \quad T \approx \mathcal{N}_{b} \mathfrak{a}_{b}^{T} ; \\
& \delta \boldsymbol{u} \approx \mathcal{N}_{a} \mathfrak{w}_{a}^{U} ; \quad \delta V \approx \mathcal{N}_{a} \mathfrak{w}_{a}^{V} ; \quad \delta T \approx \mathcal{N}_{a} \mathfrak{w}_{a}^{T} ; \\
& \nabla^{s} \boldsymbol{u} \approx \mathcal{B}_{b}^{s} \mathfrak{a}_{b}^{U} ; \quad \nabla V \approx \mathcal{B}_{b} \mathfrak{a}_{b}^{V} ; \quad \nabla T \approx \mathcal{B}_{b} \mathfrak{a}_{b}^{T} ; \\
& \nabla^{s} \delta \boldsymbol{u} \approx \mathcal{B}_{a}^{s} \mathfrak{w}_{a}^{U} ; \nabla \delta V \approx \mathcal{B}_{a} \mathfrak{w}_{a}^{V} ; \nabla \delta T \approx \mathcal{B}_{a} \mathfrak{w}_{a}^{T} ; \\
& \ddot{\boldsymbol{u}} \approx \mathcal{N}_{b} \ddot{\mathfrak{a}}_{b}^{U} ; \quad \nabla^{s} \dot{\boldsymbol{u}} \approx \mathcal{B}_{b}^{s} \dot{\mathfrak{a}}_{b}^{U} ; \quad \dot{T} \approx \mathcal{N}_{b} \dot{\mathfrak{a}}_{b}^{T}
\end{aligned}
$$

where $\mathfrak{a}$ represent the nodal values of different fields (yet called degrees of freedom), whereas $\mathfrak{w}$ represent the nodal values of their variations. In last expression, $\mathcal{N}_{a}$ denotes the standard isoparametric shape function for node $a$ (e.g., [1]), with their gradients gathered in matrix form as:

$$
\begin{aligned}
& \mathcal{B}_{a}=\nabla \mathcal{N}_{a} \\
& \mathcal{B}_{a}^{s}=\nabla^{s} \mathcal{N}_{a} .
\end{aligned}
$$

By introducing the corresponding finite element approximations into the weak form of the conservation equations, and switching from tensors to matrices by means of the Voigt notation, we can finally obtain:

$$
\left\{\begin{array}{l}
-\int_{\Omega}\left(\mathcal{B}_{a}^{s} \mathfrak{w}_{a}^{U}\right)^{\top} \boldsymbol{\sigma}+\left(\mathcal{N}_{a} \mathfrak{w}_{a}^{U}\right)^{\top}\left(\rho_{m} \mathcal{N}_{b} \ddot{\mathfrak{a}}_{b}^{U}-\boldsymbol{b}\right) \mathrm{d} \Omega+\int_{\Gamma}\left(\mathcal{N}_{a} \mathfrak{w}_{a}^{U}\right)^{\top} \overline{\boldsymbol{t}}^{c} \mathrm{~d} \Gamma=0 \\
\int_{\Omega}\left(\mathcal{B}_{a} \mathfrak{w}_{a}^{V}\right)^{\top} \boldsymbol{j} \mathrm{d} \Omega-\int_{\Gamma} \mathcal{N}_{a} \mathfrak{w}_{a}^{V} \bar{j} \mathrm{~d} \Gamma=0 \\
\int_{\Omega}\left(\mathcal{B}_{a} \mathfrak{w}_{a}^{T}\right)^{\top} \boldsymbol{q}-\mathcal{N}_{a} \mathfrak{w}_{a}^{T}\left[\rho_{m} c_{p} \mathcal{N}_{b} \dot{\mathfrak{a}}_{b}^{T}-r+\mathcal{N}_{b} \mathfrak{a}_{b}^{T} \boldsymbol{\beta}^{\top}\left(\mathcal{B}_{b}^{s} \dot{\mathfrak{a}}_{b}^{U}-\dot{\boldsymbol{\varepsilon}}^{p}\right)+\left(\boldsymbol{j}+\dot{\boldsymbol{D}}^{p}\right)^{\top} \mathcal{B}_{b} \mathfrak{a}_{b}^{V}\right. \\
\left.\quad+\left(\dot{\boldsymbol{B}}^{p}\right)^{\top} \mathcal{B}_{b} \mathfrak{a}_{b}^{\varphi}-\dot{\gamma}^{p} \sqrt{\frac{2}{3}} \sigma_{y}\right] \mathrm{d} \Omega-\int_{\Gamma} \mathcal{N}_{a} \mathfrak{w}_{a}^{T} \bar{q} \mathrm{~d} \Gamma=0
\end{array}\right.
$$

where the plastic variable values are those calculated in Section 5.2, and where we introduce the discrete approximations of dual variables and fluxes:

$$
\left\{\begin{array}{l}
\boldsymbol{\sigma}=\mathcal{C}_{(6 \times 6)}\left(\mathcal{B}_{b_{(6 \times 3)}^{s}} \mathfrak{a}_{b(3 \times 1)}^{U}-\boldsymbol{\varepsilon}^{p}\right)-\boldsymbol{\beta}\left(\mathcal{N}_{b} \mathfrak{a}_{b}^{T}-T_{0}\right)+\epsilon_{0} \mathcal{B}_{b}^{\otimes}\left(\mathfrak{a}_{b}^{V}\right)^{2}-\frac{1}{2}\left[\epsilon_{0}\left(\mathcal{B}_{b} \mathfrak{a}_{b}^{V}\right)^{\top} \mathcal{B}_{b} \mathfrak{a}_{b}^{V}\right] \\
\boldsymbol{j}=-\gamma \mathcal{B}_{b} \mathfrak{a}_{b}^{V}-\gamma \alpha \mathcal{B}_{b} \mathfrak{a}_{b}^{T} \\
\boldsymbol{q}=-\kappa \mathcal{B}_{b} \mathfrak{a}_{b}^{T}+\mathcal{N}_{b} \mathfrak{a}_{b}^{T} \alpha \boldsymbol{j}
\end{array}\right.
$$

featuring $\mathbf{1}=(1,1,1,0,0,0)^{\top}$ and the subscript $\otimes$ as the tensor multiplication into Voigt notation; in this case $\mathcal{B}_{b}^{\otimes}=\mathcal{B}_{b} \otimes \mathcal{B}_{b}$ :

$$
\mathcal{B}_{b}^{\otimes}=\left(\begin{array}{cc}
\mathcal{N}_{b, 1} & \mathcal{N}_{b, 1} \\
\mathcal{N}_{b, 2} & \mathcal{N}_{b, 2} \\
\mathcal{N}_{b, 3} & \mathcal{N}_{b, 3} \\
\mathcal{N}_{b, 1} & \mathcal{N}_{b, 2} \\
\mathcal{N}_{b, 2} & \mathcal{N}_{b, 3} \\
\mathcal{N}_{b, 1} & \mathcal{N}_{b, 3}
\end{array}\right)
$$

with $\mathcal{N}_{b, i}$ is the derivative of the shape function in the direction $i$. 
By considering that the nodal values of virtual field $(\forall \mathfrak{w})$ can be picked arbitrarily, it is possible to obtain from (51) the final set of non-linear residual equations that need to be solved:

$$
\left\{\begin{aligned}
\mathcal{R}_{a}^{U} & =-\int_{\Omega} \mathcal{B}_{a}^{s \top} \boldsymbol{\sigma}+\mathcal{N}_{a}\left(\rho_{m} \mathcal{N}_{b} \ddot{\mathfrak{a}}_{b}^{U}-\boldsymbol{b}\right) \mathrm{d} \Omega+\int_{\Gamma} \mathcal{N}_{a} \overline{\boldsymbol{t}}^{c} \mathrm{~d} \Gamma \\
\mathcal{R}_{a}^{V} & =\int_{\Omega} \mathcal{B}_{a}^{\top} \boldsymbol{j} \mathrm{d} \Omega-\int_{\Gamma} \mathcal{N}_{a} \bar{j} \mathrm{~d} \Gamma \\
\mathcal{R}_{a}^{T} & =\int_{\Omega} \boldsymbol{B}_{a}^{\top} \boldsymbol{q}-\mathcal{N}_{a}\left[\rho_{m} c_{p} \mathcal{N}_{b} \dot{\mathfrak{a}}_{b}^{T}-r+\mathcal{N}_{b} \mathfrak{a}_{b}^{T} \boldsymbol{\beta}^{\top}\left(\mathcal{B}_{b}^{s} \dot{\mathfrak{a}}_{b}^{U}-\dot{\boldsymbol{\varepsilon}}^{p}\right)+\left(\boldsymbol{j}+\dot{\boldsymbol{D}}^{p}\right)^{\top} \mathcal{B}_{b} \mathfrak{a}_{b}^{V}\right. \\
& \left.-\dot{\gamma}^{p} \sqrt{\frac{2}{3}} \sigma_{y}\right] \mathrm{d} \Omega-\int_{\Gamma} \mathcal{N}_{a} \bar{q} \mathrm{~d} \Gamma .
\end{aligned}\right.
$$

\section{Time discretization}

\subsection{Global solution step by Newmark scheme}

The Newmark scheme is used for the time discretization of the global solution step. This scheme requires two parameters $\gamma$ and $\beta$ that will determine the numerical damping and order of the scheme (e.g. [1]):

$$
\begin{aligned}
\mathfrak{a}_{b, n+1} & =\mathfrak{a}_{b, n}+\Delta t \dot{\mathfrak{a}}_{b, n}+\Delta t^{2}\left[\left(\frac{1}{2}-\beta\right) \ddot{\mathfrak{a}}_{b, n}+\beta \ddot{\mathfrak{a}}_{b, n+1}\right] ; \\
\dot{\mathfrak{a}}_{b, n+1} & =\dot{\mathfrak{a}}_{b, n}+\Delta t\left[(1-\gamma) \ddot{\mathfrak{a}}_{b, n}+\gamma \ddot{\mathfrak{a}}_{b, n+1}\right]
\end{aligned}
$$

where we denoted the time step as $\Delta t=t_{n+1}-t_{n}$. These equations, often referred to as Newmark equations, are accompanied by the residual equations enforcing the zero value at time step $n+1$, which can be explicitly written as:

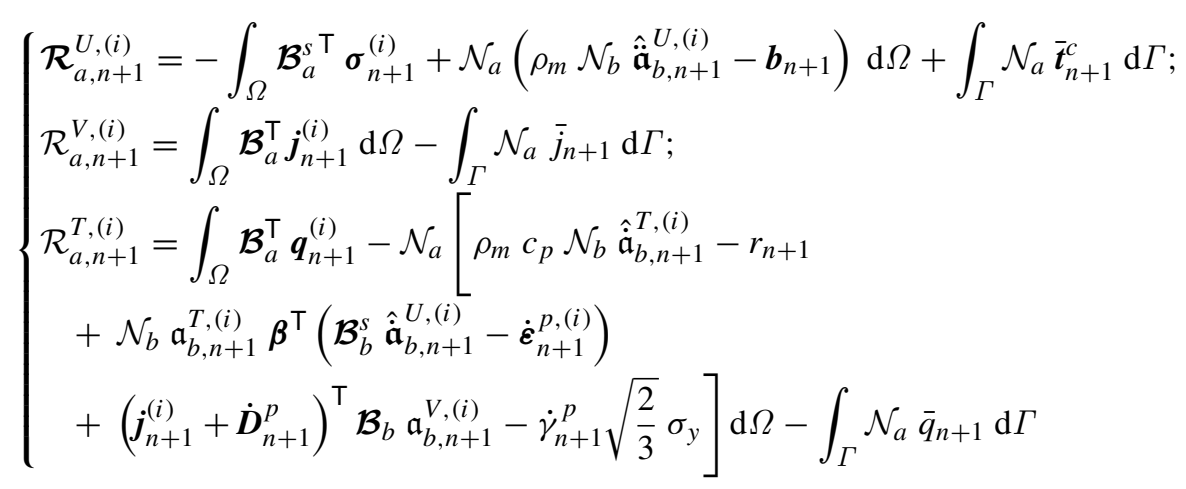

where the corresponding values for velocities $\hat{\dot{\mathfrak{a}}}_{n+1}^{(i)}$ and accelerations $\hat{\mathfrak{\mathfrak { a }}}_{n+1}^{(i)}$ are obtained by recasting the result (55) resulting with:

$$
\begin{aligned}
& \hat{\mathfrak{a}}_{b, n+1}^{(i)}=\dot{\mathfrak{a}}_{b, n}+\Delta t\left[(1-\gamma) \ddot{\mathfrak{a}}_{b, n}+\gamma \ddot{\mathfrak{a}}_{b, n+1}^{(i)}\right] \\
& \hat{\mathfrak{a}}_{b, n+1}^{(i)}=\frac{\mathfrak{a}_{b, n+1}^{(i)}-\mathfrak{a}_{b, n}-\Delta t \dot{\mathfrak{a}}_{b, n}}{\beta \Delta t^{2}}+\left(1-\frac{1}{2 \beta}\right) \ddot{\mathfrak{a}}_{b, n}
\end{aligned}
$$

and the plastic variables can be discretized by the backward Euler scheme:

$$
\begin{aligned}
& \dot{\boldsymbol{\varepsilon}}_{n+1}^{p}=\frac{\boldsymbol{\varepsilon}_{n+1}^{p}-\boldsymbol{\varepsilon}_{n}^{p}}{\Delta t} ; \\
& \dot{\boldsymbol{D}}_{n+1}^{p}=\frac{\boldsymbol{D}_{n+1}^{p}-\boldsymbol{D}_{n}^{p}}{\Delta t} ; \\
& \dot{\gamma}_{n+1}^{p}=\frac{\gamma_{n+1}^{p}}{\Delta t} .
\end{aligned}
$$


Thus, the time stepping scheme of this kind will finally render the set of nonlinear algebraic equations. To solve such a nonlinear problem, we use Newton's iterative method where at each iteration $(i+1)$ we perform the consistent linearization of residual leading to:

$$
\boldsymbol{\mathcal { R }}_{a, n+1}^{(i+1)}=0 \quad \Rightarrow \quad \boldsymbol{\mathcal { R }}_{a, n+1}^{(i)}+\left.\frac{\partial \boldsymbol{\mathcal { R }}_{a, n+1}}{\partial \mathfrak{a}_{b, n+1}}\right|^{(i)} \Delta \mathfrak{a}_{b, n+1}^{(i)}=0 ; \mathcal{R}_{a, n+1}^{(i)}=\left[\mathcal{R}^{U}, \mathcal{R}^{V}, \mathcal{R}^{T}\right]_{a, n+1}^{\top,(i)}
$$

where $\Delta \mathfrak{a}_{b}$ are iterative contributions to nodal values of temperature, electric field, along with displacements, velocities and accelerations. At each iterative sweep, we can then perform the corresponding state variable updates according to:

$$
\mathfrak{a}_{b, n+1}^{(i+1)}=\mathfrak{a}_{b, n+1}^{(i)}+\Delta \mathfrak{a}_{b, n+1}^{(i)} .
$$

In the first iteration within each time step we will take the starting guess equal to the converged value at the previous step:

$$
\mathfrak{a}_{b, n+1}^{(0)}=\mathfrak{a}_{b, n} .
$$

The mechanics part of the residual vector at particular iteration can be further compressed, reducing it to the form presented explicitly in (56). Namely, the first term in (59) can be reduced to so-called effective tangent stiffness for mechanical part, which is directly used to compute the iterative contributions to displacement increments:

$$
\mathcal{S}_{a b}^{U,(i)}=-\left.\frac{\partial \mathcal{R}_{a}^{U}}{\partial \mathfrak{a}_{b}}\right|^{(i)}-\left.\frac{\partial \mathcal{R}_{a}^{U}}{\partial \dot{\mathfrak{a}}_{b}} \frac{\partial \dot{\mathfrak{a}}_{b}}{\partial \mathfrak{a}_{b}}\right|^{(i)}-\left.\frac{\partial \mathcal{R}_{a}^{U}}{\partial \ddot{\mathfrak{a}}_{b}} \frac{\partial \ddot{\mathfrak{a}}_{b}}{\partial \mathfrak{a}_{b}}\right|^{(i)}
$$

where time-step subscript $n+1$ was dropped to simplify notation. By exploiting the relations between the nodal displacements and its first and second derivatives provided by the Newmark scheme, we can provide the closed form final linearized problem to be solved. More precisely, in view of the Newmark result for constructing discrete approximations for nodal velocities and accelerations in (55), we can write:

$$
\frac{\partial \ddot{\mathfrak{a}}_{b}}{\partial \mathfrak{a}_{b}}=\frac{1}{\beta \Delta t^{2}} ; \quad \frac{\partial \dot{\mathfrak{a}}_{b}}{\partial \mathfrak{a}_{b}}=\frac{\partial \dot{\mathfrak{a}}_{b}}{\partial \ddot{\mathfrak{a}}_{b}} \frac{\partial \ddot{\mathfrak{a}}_{b}}{\partial \mathfrak{a}_{b}}=\frac{\gamma}{\beta \Delta t} .
$$

Thus, the final form of the tangent operator for mechanics part can now be written as:

$$
\mathcal{S}_{a b}^{U,(i)}=\mathcal{K}_{a b}^{(i)}+\frac{\gamma}{\beta \Delta t} \mathcal{D}_{a b}^{(i)}+\frac{1}{\beta \Delta t^{2}} \mathcal{M}_{a b}^{(i)}
$$

where $\mathcal{K}_{a b}=-\frac{\partial \mathcal{R}_{a}}{\partial \mathfrak{a}_{b}}$ is the elastoplastic tangent stiffness matrix, $\mathcal{D}_{a b}=-\frac{\partial \mathcal{R}_{a}}{\partial \dot{\mathfrak{a}}_{b}}$ is the damping matrix and $\mathcal{M}_{a b}=-\frac{\partial \boldsymbol{\mathcal { R }}_{a}}{\partial \ddot{\mathfrak{a}}_{b}}$ is the mass matrix.

Given the solution for displacement increment at iteration $(i)$, we proceed to compute the displacement updates $\mathfrak{a}_{n+1}^{(i+1)}$ by using the result in (60).

\subsection{Local iterative solution for plasticity with isotropic hardening}

This local computation has to be performed for every Gauss point in agreement with the corresponding result $\mathfrak{a}_{n+1}^{(i)}$ obtained in the global phase. For proposed generalization of von Mises plasticity, we first define the yield function for coupled problem of this kind:

$$
\left.\phi_{n+1}\right|_{\mathfrak{a}_{n+1}^{(i)}}=\left\|\operatorname{dev}\left(\boldsymbol{\sigma}_{n+1}\right)\right\|-\sqrt{\frac{2}{3}}\left(\sigma_{y}\left(T_{n+1}, \boldsymbol{E}_{n+1}\right)-q_{n+1}\right) .
$$


The elastic trial step is computed first, with the zero value of plastic multiplier $\gamma_{n+1}^{p \text {,tr }}=0$. This result with:

$$
\begin{aligned}
\boldsymbol{\varepsilon}_{n+1}^{p, \operatorname{tr}} & =\boldsymbol{\varepsilon}_{n}^{p} ; \\
\zeta_{n+1}^{\operatorname{tr}} & =\zeta_{n} ; \\
s_{n+1}^{p, \operatorname{tr}} & =s_{n}^{p} ; \\
\boldsymbol{D}_{n+1}^{p, \operatorname{tr}} & =\boldsymbol{D}_{n}^{p} .
\end{aligned}
$$

These results are exploited in computation of the corresponding trial values of stress and hardening stress-like variable:

$$
\begin{aligned}
& \boldsymbol{\sigma}_{n+1}^{\operatorname{tr}}=\mathcal{C}\left(\boldsymbol{\varepsilon}_{n+1}-\boldsymbol{\varepsilon}_{n}^{p}\right)-\boldsymbol{\beta}\left(T_{n+1}-T_{0}\right)+\epsilon \boldsymbol{E}_{n+1}^{\otimes}-\frac{\mathbf{1}}{2} \boldsymbol{E}_{n+1} \cdot \boldsymbol{E}_{n+1} ; \\
& q_{n+1}^{\mathrm{tr}}=q_{n}=-K \zeta_{n} .
\end{aligned}
$$

To ensure that the elastic step is acceptable, the trial value of yield function must remain negative or zero

$$
0 \geq \phi_{n+1}^{\mathrm{tr}}=\left\|\operatorname{dev}\left(\boldsymbol{\sigma}_{n+1}^{\mathrm{tr}}\right)\right\|-\sqrt{\frac{2}{3}}\left(\sigma_{y}\left(T_{n+1}, \boldsymbol{E}_{n+1}\right)-q_{n+1}^{\mathrm{tr}}\right) .
$$

If such a condition is not verified, the step is plastic. The latter implies that all the internal variables must be recomputed by integrating the evolution equations in (46):

$$
\begin{aligned}
& \boldsymbol{\varepsilon}_{n+1}^{p}=\boldsymbol{\varepsilon}_{n}^{p}+\gamma_{n+1}^{p} \boldsymbol{v}_{n+1} ; \\
& \zeta_{n+1}=\zeta_{n}+\sqrt{\frac{2}{3}} \gamma_{n+1}^{p} ; \\
& s_{n+1}^{p}=s_{n}^{p}+\gamma_{n+1}^{p} \sqrt{\frac{2}{3}} \omega_{\mathrm{t}}\left(\sigma_{y_{0}}+K_{0} \zeta_{n+1}\right) ; \\
& \boldsymbol{D}_{n+1}^{p}=\boldsymbol{D}_{n}^{p}+\gamma_{n+1}^{p} \frac{\boldsymbol{E}}{\|\boldsymbol{E}\|} \sqrt{\frac{2}{3}} \omega_{\mathrm{e}}\left(\sigma_{y_{0}}+K_{0} \zeta_{n+1}\right) .
\end{aligned}
$$

The plastically admissible value of stresses can be computed as:

$$
\begin{aligned}
& \boldsymbol{\sigma}_{n+1}=\boldsymbol{\sigma}_{n+1}^{\mathrm{tr}}-\gamma_{n+1}^{p} \mathcal{C} \boldsymbol{v}_{n+1} \\
& q_{n+1}=-K \zeta_{n+1} .
\end{aligned}
$$

We note that the final value of plastic multiplier $\gamma_{n+1}^{p}$ can be obtained from $\phi_{n+1}=0$, by taking into account that:

$$
\begin{aligned}
& \boldsymbol{v}_{n+1}=\boldsymbol{v}_{n+1}^{\mathrm{tr}} ; \\
& \left\|\operatorname{dev}\left(\boldsymbol{\sigma}_{n+1}\right)\right\|=\left\|\operatorname{dev}\left(\boldsymbol{\sigma}_{n+1}^{\mathrm{tr}}\right)\right\|-2 \mu \gamma_{n+1}^{p}
\end{aligned}
$$

where $\mu$ is the shear modulus. We finally obtain:

$$
\gamma_{n+1}^{p}=\frac{\phi_{n+1}^{\mathrm{tr}}}{2 \mu+\frac{2 K}{3}} .
$$

\subsection{Consistent tangent thermo-electro-elastoplastic modulus}

The computation of the consistent tangent thermo-electro-elastoplastic modulus is carried by generalizing the scheme first proposed for classical plasticity in [19]. At converged values of local computation, we take a new iterative sweep brought by an increment of total strain. The corresponding linearized form of local problem is obtained in terms 
of dual variables:

$$
\mathcal{L}_{n+1}^{(k)}\left(\begin{array}{c}
\Delta \boldsymbol{\sigma}_{n+1} \\
\Delta T_{n+1} \\
\Delta \boldsymbol{E}_{n+1} \\
\Delta \gamma_{n+1}^{p} \\
\Delta q_{n+1}
\end{array}\right)=\left(\begin{array}{c}
-\boldsymbol{\varepsilon}_{n+1}^{e}+\boldsymbol{\varepsilon}_{n}^{e}+\Delta \boldsymbol{\varepsilon}_{n+1}^{(i)}-\gamma_{n+1}^{p} \frac{\partial \phi}{\partial \boldsymbol{\sigma}_{n+1}} \\
-s_{n+1}^{e}+s_{n}^{e}+\Delta s_{n+1}^{(i)}-\gamma_{n+1}^{p} \frac{\partial \phi}{\partial T_{n+1}} \\
-\boldsymbol{D}_{n+1}^{e}+\boldsymbol{D}_{n}^{e}+\Delta \boldsymbol{D}_{n+1}^{(i)}-\gamma_{n+1}^{p} \frac{\partial \phi}{\partial \boldsymbol{E}_{n+1}} \\
\phi_{n+1} \\
\zeta_{n+1}-\zeta_{n}-\gamma_{n+1}^{p} \frac{\partial \phi}{\partial q_{n+1}}
\end{array}\right)
$$

where the tangent matrix is, for the most general case:

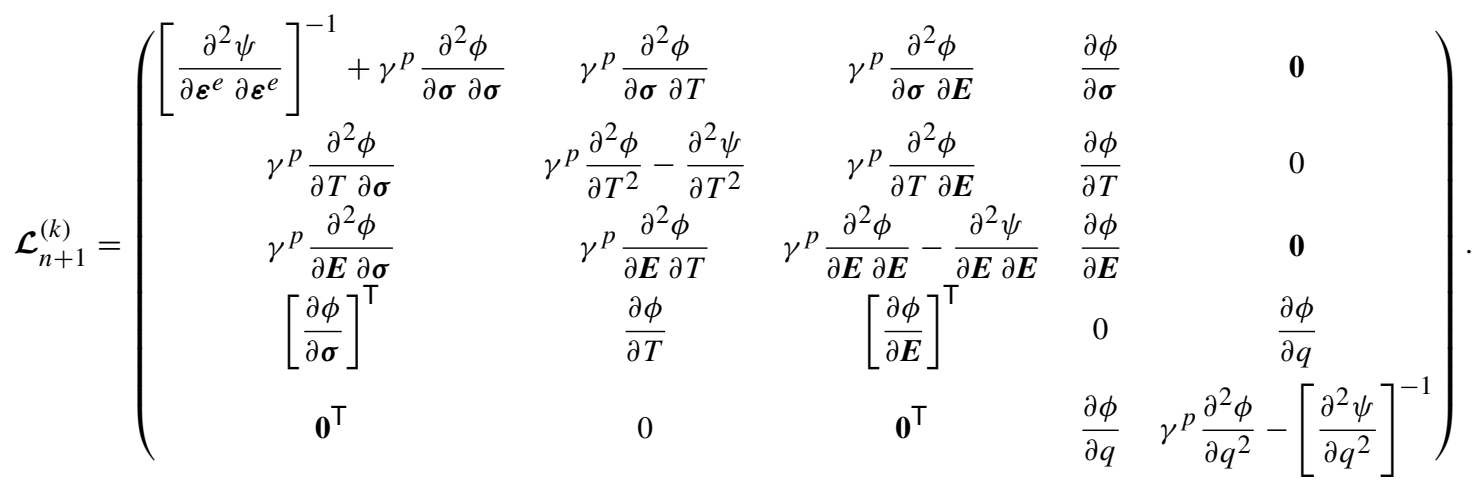

The subsequent iterative values can then be obtained with simple additive updates:

$$
\begin{aligned}
& \boldsymbol{\sigma}_{n+1}^{(k+1)}=\boldsymbol{\sigma}_{n}^{(k)}+\Delta \boldsymbol{\sigma}_{n+1} \\
& T_{n+1}^{(k+1)}=T_{n}^{(k)}+\Delta T_{n+1} \\
& \boldsymbol{E}_{n+1}^{(k+1)}=\boldsymbol{E}_{n}^{(k)}+\Delta \boldsymbol{E}_{n+1} \\
& \gamma_{n+1}^{p,(k+1)}=\gamma_{n}^{p,(k)}+\Delta \gamma_{n+1}^{p} \\
& q_{n+1}^{(k+1)}=q_{n}^{(k)}+\Delta q_{n+1} .
\end{aligned}
$$

With the converged values of internal variables in the local computation phase, we can write the result in (73) as:

$$
\mathcal{L}_{n+1}\left(\begin{array}{c}
\Delta \boldsymbol{\sigma}_{n+1} \\
\Delta T_{n+1} \\
\Delta \boldsymbol{E}_{n+1} \\
\gamma_{n+1}^{p} \\
q_{n+1}-q_{n}
\end{array}\right)=\left(\begin{array}{c}
\Delta \boldsymbol{\varepsilon}_{n+1}^{(i+1)} \\
\Delta s_{n+1}^{(i+1)} \\
\Delta \boldsymbol{D}_{n+1}^{(i)} \\
0 \\
0
\end{array}\right)
$$

Further, by using the static condensation method (e.g. [1]) we can reduce the size of this system:

$$
\left(\begin{array}{lll}
\mathcal{L}_{a a}-\mathcal{L}_{a b} \mathcal{L}_{b b}^{-1} & \mathcal{L}_{b a}
\end{array}\right)\left(\begin{array}{c}
\Delta \boldsymbol{\sigma} \\
\Delta T \\
\Delta \boldsymbol{E}
\end{array}\right)_{n+1}=\left(\begin{array}{c}
\Delta \boldsymbol{\varepsilon} \\
\Delta s \\
\Delta \boldsymbol{D}
\end{array}\right)_{n+1}^{(i+1)}
$$


where:

$$
\begin{aligned}
\mathcal{L}_{a a_{(10 \times 10)}}:= & \left(\begin{array}{ccc}
{\left[\frac{\partial^{2} \psi}{\partial \boldsymbol{\varepsilon}^{e} \partial \boldsymbol{\varepsilon}^{e}}\right]^{-1}+\gamma^{p} \frac{\partial^{2} \phi}{\partial \boldsymbol{\sigma} \partial \boldsymbol{\sigma}}} & \gamma^{p} \frac{\partial^{2} \phi}{\partial \boldsymbol{\sigma} T} & \gamma^{p} \frac{\partial^{2} \phi}{\partial \boldsymbol{\sigma} \partial \boldsymbol{E}} \\
\gamma^{p} \frac{\partial^{2} \phi}{\partial T \partial \boldsymbol{\sigma}} & \gamma^{p} \frac{\partial^{2} \phi}{\partial T^{2}}-\frac{\partial^{2} \psi}{\partial T^{2}} & \gamma^{p} \frac{\partial^{2} \phi}{\partial T \partial \boldsymbol{E}} \\
\gamma^{p} \frac{\partial^{2} \phi}{\partial \boldsymbol{E} \partial \boldsymbol{\sigma}} & \gamma^{p} \frac{\partial^{2} \phi}{\partial \boldsymbol{E} \partial T} & \gamma^{p} \frac{\partial^{2} \phi}{\partial \boldsymbol{E} \partial \boldsymbol{E}}-\frac{\partial^{2} \psi}{\partial \boldsymbol{E} \partial \boldsymbol{E}}
\end{array}\right) ; \\
\mathcal{L}_{a b_{(10 \times 2)}}:= & \left(\begin{array}{cc}
\frac{\partial \phi}{\partial \boldsymbol{\sigma}} & \mathbf{0} \\
\frac{\partial \phi}{\partial T} & 0 \\
\frac{\partial \phi}{\partial \boldsymbol{E}} & \mathbf{0}
\end{array}\right) ; \quad \mathcal{L}_{b b_{(2 \times 2)}}:=\left(\begin{array}{cc}
0 & \frac{\partial \phi}{\partial q} \\
\frac{\partial \phi}{\partial q} & \left.\gamma^{p} \frac{\partial^{2} \phi}{\partial q^{2}}-\left[\frac{\partial^{2} \psi}{\partial q^{2}}\right]^{-1}\right) ; \quad \mathcal{L}_{b a_{(2 \times 10)}}
\end{array}\right):=\mathcal{L}_{a b}^{\top} .
\end{aligned}
$$

Due to the zero column and row of $\mathcal{L}_{a b}$ and $\mathcal{L}_{b a}$ respectively, the only relevant term of $\mathcal{L}_{b b}^{-1}$ for the multiplication in (77) is the first one:

$$
\hat{L}:=-\mathcal{L}_{b b}^{-1}(1,1)=\frac{b}{a^{2}}
$$

where:

$$
a:=\mathcal{L}_{b b}(1,2)=\mathcal{L}_{b b}(2,1)=\frac{\partial \phi}{\partial q} ; \quad b:=\mathcal{L}_{b b}(2,2)=\gamma^{p} \frac{\partial^{2} \phi}{\partial q^{2}}-\left[\frac{\partial^{2} \psi}{\partial q^{2}}\right]^{-1}
$$

so that this multiplication is simplified to:

$$
-\mathcal{L}_{a b} \mathcal{L}_{b b}^{-1} \mathcal{L}_{b a}=\hat{L} \xi \xi^{\top}
$$

where $\boldsymbol{\xi}$ is a vector containing the first column of $\mathcal{L}_{a b}$. By substituting (81) into (77), after some further simplifications, we obtain:

$$
\underbrace{\left(\mathcal{L}_{a a}+\hat{L} \xi \xi^{\top}\right)}_{\hat{\mathcal{C}}}\left(\begin{array}{c}
\Delta \boldsymbol{\sigma} \\
\Delta T \\
\Delta \boldsymbol{E}
\end{array}\right)_{n+1}=\left(\begin{array}{c}
\Delta \boldsymbol{\varepsilon} \\
\Delta s \\
\Delta \boldsymbol{D}
\end{array}\right)_{n+1}^{(i+1)}
$$

featuring $\hat{\mathcal{C}}$ as a consistent tangent thermo-electro-elastoplastic modulus for the given variables. By exchanging the roles between variables $\boldsymbol{\sigma}$ and $\boldsymbol{\varepsilon}$, we can obtain the final form of tangent modulus for the global phase $\overline{\mathcal{C}}$; namely, with the split of $\hat{\mathcal{C}}$ :

$$
\hat{\mathcal{C}}=\left(\begin{array}{ll}
\hat{\mathcal{C}}_{a a_{(6 \times 6)}} & \hat{\mathcal{C}}_{a b_{(6 \times 7)}} \\
\hat{\mathcal{C}}_{b a_{(7 \times 6)}} & \hat{\mathcal{C}}_{b b_{(7 \times 7)}}
\end{array}\right)
$$

we can easily construct $\overline{\mathcal{C}}$ which can be written as:

$$
\overline{\mathcal{C}}=\left(\begin{array}{cc}
\hat{\mathcal{C}}_{a a_{(6 \times 6)}}^{-1} & {\left[-\hat{\mathcal{C}}_{a a}^{-1} \hat{\mathcal{C}}_{a b}\right]_{(6 \times 4)}} \\
{\left[\hat{\mathcal{C}}_{b a} \hat{\mathcal{C}}_{a a}^{-1}\right]_{(4 \times 6)}} & {\left[\hat{\mathcal{C}}_{b b}-\hat{\mathcal{C}}_{b a} \hat{\mathcal{C}}_{a a}^{-1} \hat{\mathcal{C}}_{a b}\right]_{(4 \times 4)}}
\end{array}\right) \Rightarrow \overline{\mathcal{C}}\left(\begin{array}{c}
\Delta \boldsymbol{\varepsilon} \\
\Delta T \\
\Delta \boldsymbol{E}
\end{array}\right)_{n+1}=\left(\begin{array}{c}
\Delta \boldsymbol{\sigma} \\
\Delta s \\
\Delta \boldsymbol{D}
\end{array}\right)_{n+1}
$$

\section{Numerical examples}

In this section, the results of numerical simulations are presented for plasticity problem with thermo-electromechanic coupling of this kind, including the practical applications in terms of Peltier cells. All computations are performed by a research version of the well-known computer code FEAP [20]. 
Table 2

Properties of the material used in validation example.

\begin{tabular}{lll}
\hline Property & Units & Value \\
\hline Young modulus $E$ & $\mathrm{GPa}$ & 200 \\
Mass density $\rho_{m}$ & $\mathrm{~kg} / \mathrm{m}^{3}$ & $7.8 \times 10^{3}$ \\
Specific heat $c_{p}$ & $\mathrm{~J} / \mathrm{kg} \mathrm{K}$ & $1.2 \times 10^{6}$ \\
Seebeck coefficient $\alpha$ & $\mathrm{V} / \mathrm{K}$ & $2 \times 10^{-4}$ \\
Thermal expansion coef. $\alpha_{T}$ & $1 / \mathrm{K}$ & $1.5 \times 10^{-5}$ \\
Thermal conductivity $\kappa$ & $\mathrm{W} / \mathrm{K} \mathrm{m}$ & 0.15 \\
Electric conductivity $\gamma$ & $\mathrm{A} / \mathrm{V} \mathrm{m}$ & $10^{6}$ \\
\hline
\end{tabular}

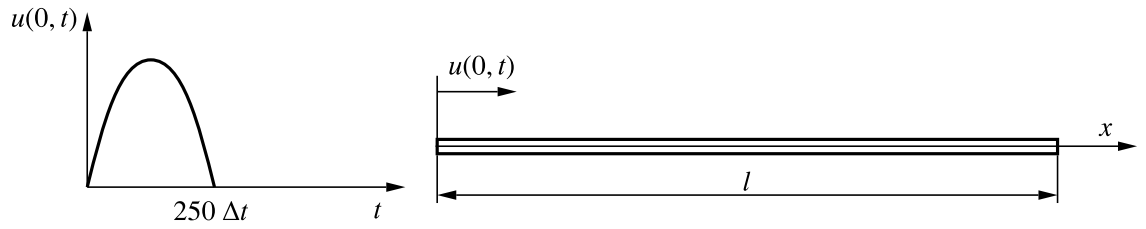

Fig. 1. Scheme of the geometry considered and the pulse applied to the left end.

\subsection{Electro-thermo-mechanical coupling for transient loading}

In this first example we consider a transient problem where different coupling effects are easier to validate, thus providing the sort of benchmark result. Namely, we compute an electro-thermo-mechanical coupling in 3D domain in the form of a bar, with one dimension significantly bigger than the other two. The boundary conditions are chosen in agreement with propagation along the bar with a displacement time variation imposed at the left end while the right end of the bar is free; we also impose adiabatic boundary for the thermal and isolated contour for the electromagnetic field (see Fig. 1). The chosen material properties are shown in Table 2. The choice of Newmark scheme parameters $\gamma=1.5$ and $\beta=1$ results with the highest numerical dissipation (see [21]).

The bar is set in motion by a displacement pulse imposed on the left end (see Fig. 1) that propagates through the bar, with its period being much lower than the one corresponding to inertia of the bar. This displacement produces the corresponding local change in the time derivative of the strain, which further generates the structural heating defined in (39) and leads to a temperature change. These temperature changes also lead to change in the electric field, which is affected through the constitutive behavior of the material (23) assuming $\boldsymbol{j}=\mathbf{0}$ due to the isolated boundary conditions.

In Fig. 2 we present the computed response for the displacement in the bar longitudinal direction, the electric voltage and the temperature for the point in the middle of the bar $x=l / 2$ through the transient sequence. The time scale used is the step size $\Delta t=5 \times 10^{-8} \mathrm{~s}$.

Regarding the evolution of the mechanical field, this problem can be seen as a wave propagation. When the wave arrives to the right end, the reflected wave keeps the same sign as the wave that originally came, and when it arrives to the left side the sign changes due to the restricted movement. This problem is considered in elastic regime so there is no plastic dissipation. Nonetheless, the maximum displacement is gradually reduced due to dissipation by conduction, which is produced for non-homogenize temperature distribution along the bar. Moreover, the wave length is increased in the course of this wave propagation.

The evolution of other two fields is directly affected by the evolution of displacement field. The temperature in particular is proportional to the time derivative of the strain or strain rate, which changes the sign within the displacement pulse. Moreover, when the wave passes by the middle point, the strain will be positive or negative, which depends upon the direction of the wave and the sign of the displacement. As it can be seen in Fig. 2, a wave passing from left to right in the ascending part generates at first a positive strain rate, but once passed the maximum displacement, the same wave produces negative strain rate. The voltage evolution is directly affected by the evolution of the temperature, but with the sign changed as stated in (23). 

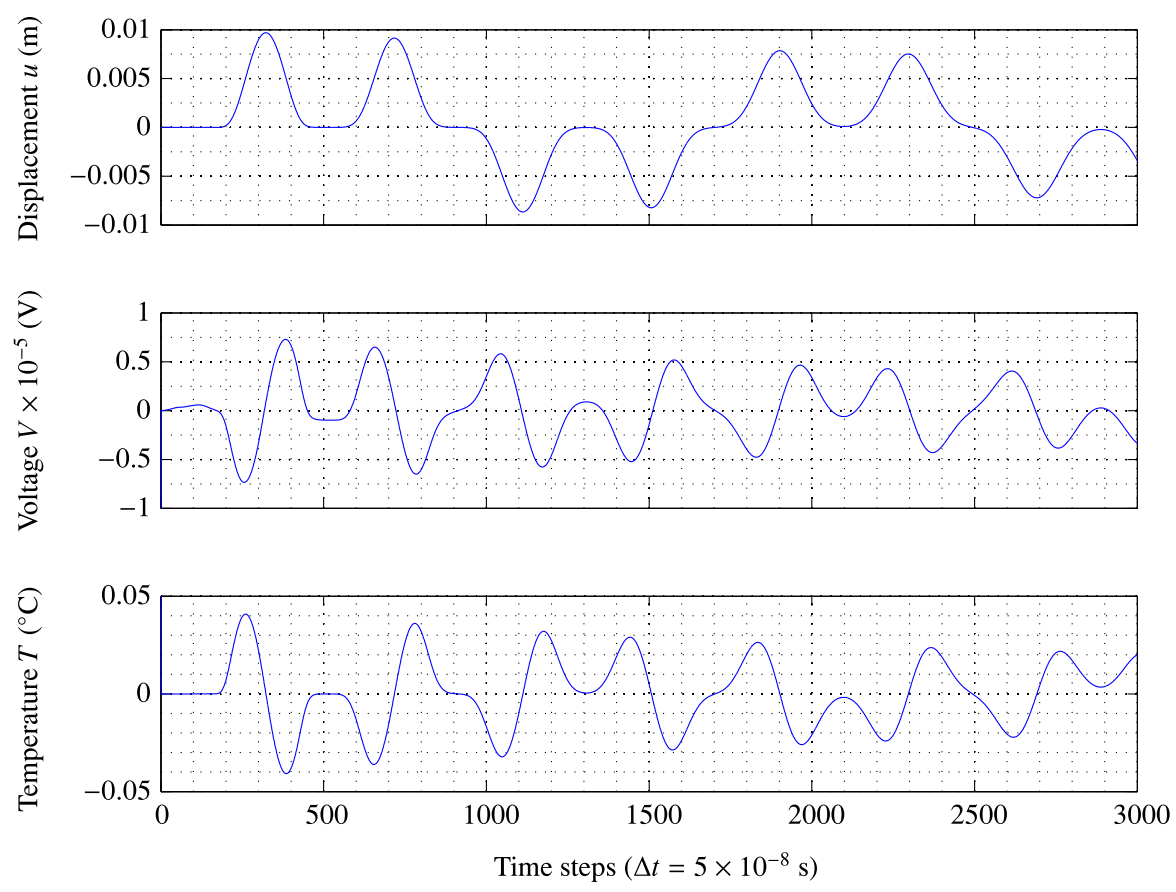

Fig. 2. Computed displacement, voltage and temperature in the bar middle point $x=l / 2$.

Table 3

Material properties for every material used in the Peltier Cell.

\begin{tabular}{|c|c|c|c|c|c|}
\hline Property & Units & Alumina & Copper & Tin-Lead & $\mathrm{Bi}_{2} \mathrm{Te}_{3}$ \\
\hline Hardening modulus $K$ & $\mathrm{GPa}$ & 5 & 5 & 5 & 1 \\
\hline Yield stress $\sigma_{y}$ & MPa & 15,400 & 100 & 180 & 55 \\
\hline Mass density $\rho$ & $\mathrm{kg} / \mathrm{m}^{3}$ & 3570 & 8960 & 7310 & 7530 \\
\hline Specific heat $c_{p}$ & $\mathrm{~J} / \mathrm{kg} \mathrm{K}$ & 837 & 385 & 226 & 544 \\
\hline Expansion coefficient $\alpha_{T}$ & $1 /{ }^{\circ} \mathrm{C}$ & $5.00 \times 10^{-6}$ & $1.70 \times 10^{-5}$ & $2.70 \times 10^{-5}$ & $1.68 \times 10^{-5}$ \\
\hline First Lamé parameter $\lambda^{I}$ & $\mathrm{~N} / \mathrm{m}^{2}$ & $1.63 \times 10^{11}$ & $7.16 \times 10^{10}$ & $3.25 \times 10^{10}$ & $6.71 \times 10^{10}$ \\
\hline Shear stress $\mu$ & $\mathrm{N} / \mathrm{m}^{2}$ & $1.51 \times 10^{11}$ & $4.39 \times 10^{10}$ & $1.68 \times 10^{10}$ & $1.68 \times 10^{10}$ \\
\hline Thermal conductivity $\kappa$ & $\mathrm{W} / \mathrm{K} \mathrm{m}$ & 35.3 & 386 & 48 & $\kappa(T)$ \\
\hline Electric conductivity $\gamma$ & $\mathrm{A} / \mathrm{V} \mathrm{m}$ & 0 & $5.81 \times 10^{7}$ & $4.72 \times 10^{6}$ & $\gamma(T)$ \\
\hline Seebeck coefficient $\alpha$ & $\mathrm{V} / \mathrm{K}$ & 0 & 0 & 0 & $\alpha(T)$ \\
\hline
\end{tabular}

\subsection{Peltier cells with stress evolution under plasticity constraint}

In this numerical example, we simulate the behavior of half a thermocouple of a pulsed Peltier cell, representing a cooler based on the Peltier effect under the action of an electric flux. This cell consists of four different materials: thermoelectric material (here chosen $\mathrm{Bi}_{2} \mathrm{Te}_{3}$ ), copper, alumina and a tin-lead solder.

A complete simulation for elastic regime can be found in [18]. The material properties for this problem are presented in Table 3. Moreover, some of these properties for the $\mathrm{Bi}_{2} \mathrm{Te}_{3}$ are chosen as temperature dependent:

$$
\begin{aligned}
& \alpha(T)=1.988 \times 10^{-4}+3.353 \times 10^{-7} T+7.52 \times 10^{-10} T^{2} \\
& \kappa(T)=1.663-3.58 \times 10^{-3} T+3.195 \times 10^{-5} T^{2} \\
& \gamma(T)=1.096 \times 10^{5}-5.59 \times 10^{2} T+2.498 T^{2} .
\end{aligned}
$$

Finally, the alumina is considered to remain elastic material (setting very high value for yield stress) because the plasticity begins well after the ultimate stress is reached. The same values for the Newmark parameters are chosen as in the last example. 

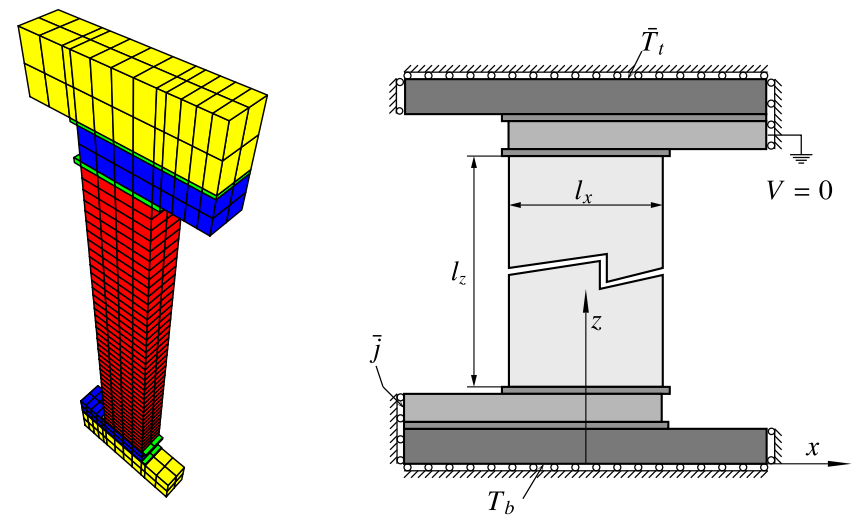

Fig. 3. Left, mesh used in this numerical example, each material represented in a different color: $\mathrm{Bi}_{2} \mathrm{Te}_{3}$ in red, copper in blue, solder in green and alumina in yellow. Right, boundary conditions imposed in this problem. (For interpretation of the references to color in this figure legend, the reader is referred to the web version of this article.)

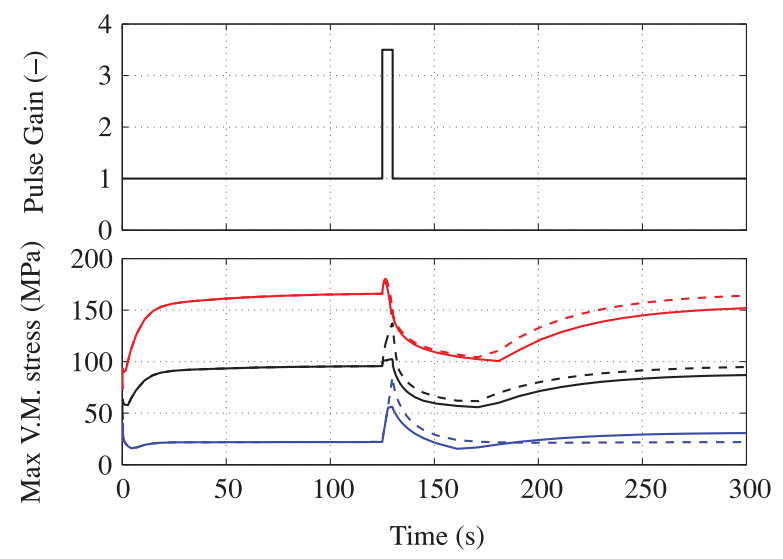

Fig. 4. Maximum von Mises stress for solder (red), copper (black) and $\mathrm{Bi}_{2} \mathrm{Te}_{3}$ (blue) when an electric square pulse of gain $P=3.5$ and duration $\Delta t_{p}=5 \mathrm{~s}$ is applied at $t=125 \mathrm{~s}$. Full line, computed result with elastoplastic response; dashed line, computed results for elastic response. (For interpretation of the references to color in this figure legend, the reader is referred to the web version of this article.)

The finite element mesh constructed with the hexahedral 8-node finite elements used for computing the solution to this problem is shown in Fig. 3. Different materials are presented in different colors. The boundary conditions are shown in Fig. 3 right. We fix the displacements in directions $x$ and $z$ at the respective end faces, along with the symmetry condition in the plane $y=0$. The temperature is prescribed at the top face $\bar{T}_{t}=50{ }^{\circ} \mathrm{C}$ (called hot side) along with a zero heat flux imposed at the bottom (cold side) where the temperature $T_{b}$ is left free. The electric flux is introduced at the bottom left end of the copper while ground voltage is assumed at the top right end copper.

The applied loading is presented in Fig. 4 top. First, an electric flux $\boldsymbol{j}_{s s}=I_{o p} / A$ is introduced, where $I_{o p}$ is an optimal electric current that maximizes the temperature difference $\bar{T}_{t}-T_{b}$ in steady-state (more details in [18]) with A as the transversal area of the copper. This electric flux value is kept fixed until evolution for all the degrees of freedom has been stabilized, and a steady-state has been reached. After this moment, the flux is incremented $P$ times, where $P=\boldsymbol{j} / \boldsymbol{j}_{s s}$ is the pulse gain, which implies automatically that $T_{b}$ decreases until a minimum is reached, but with an overheating penalty reached right afterwards. The steady-state temperature is reached eventually after reimposing $P=1$ if no other pulse is introduced.

Fig. 4 provides the representation of the maximum von Mises stress values calculated in Gauss points for each material in both elastic and plastic regimes. In the steady-state, the maximum stresses are similar since the yield stress has not been reached yet. However, with the increase of electric flux, the stress increments seen in [18] due to accumulation of Joule heating, are no longer the same. 

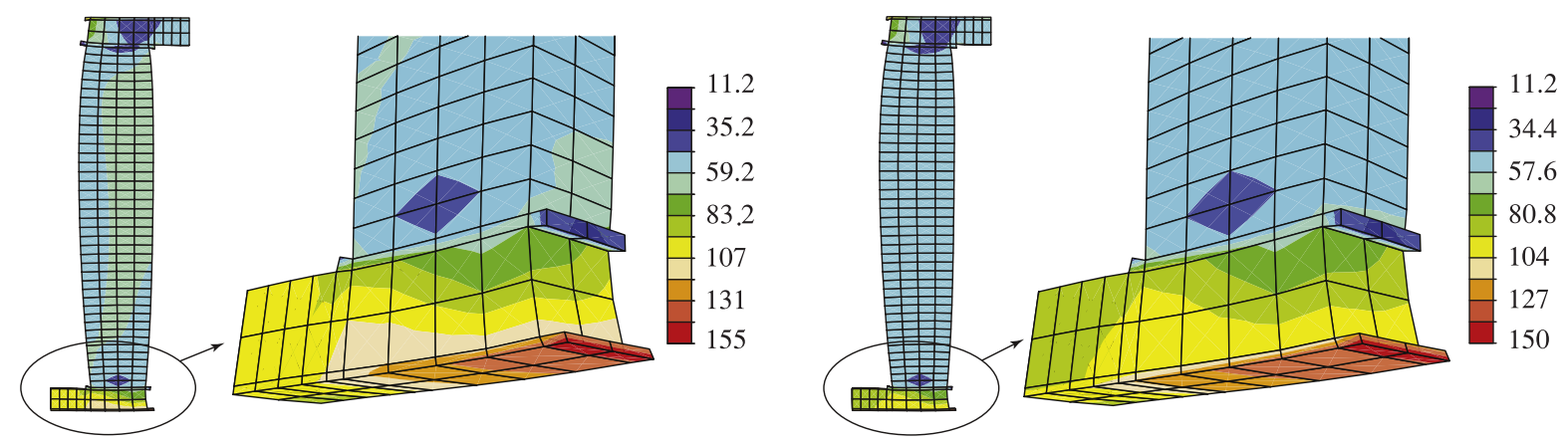

Fig. 5. Von Mises stress [MPa] contour in deformed configuration for elastic (left) and plastic regimes (right). All materials but the alumina. Detailed zoom at the bottom side of the Peltier cell.

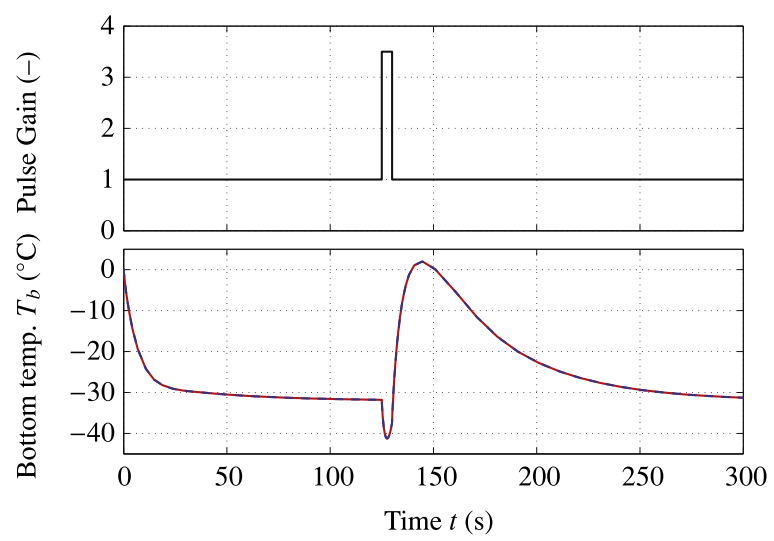

Fig. 6. Top: electric flux pulse gain introduced in the problem. Bottom: temperature at the cold side for elastic (red, full) and plastic regime (blue, dashed). (For interpretation of the references to color in this figure legend, the reader is referred to the web version of this article.)

When the pulse is introduced reaching the yield stress $\sigma_{y}$, the subsequent stress-rate increase is reduced, due to the corresponding increase in plastic strains. These changes result in a noted decrease of the maximum stresses computed with activated plasticity constraint compared to the elastic case, specially in the copper and in the thermoelectric material. Once the steady-state gain is restated, the asymptotic stresses are reduced as a result of the plastic deformation in the copper and the solder. Moreover, due to the stresses redistribution, enforced by plasticity constraint, the stresses are increased in the $\mathrm{Bi}_{2} \mathrm{Te}_{3}$. Thus, a new study of the introduction of another pulse after the application of the first is needed.

In Fig. 5 the nodal von Mises projection distribution is shown at the end of the pulse $t=130 \mathrm{~s}$, when the maximum stresses take place, along with a detailed zoom of the cold side, the most stressed part of the structure. The alumina has been omitted in order to show more clearly the differences with respect to the plastic materials.

In both cases the maximum is located at the bottom since this side has a bigger difference with respect to the reference temperature than the hot side. The maximum values are rather similar in both regimes. This is due to the influence of alumina that remains elastic, which stabilizes the corresponding stress distribution inside with no marked changes.

The first noticeable difference is that the stress distribution of the lower values is quite different. This can be appreciated specially for the copper, where the part on the bottom left has quite reduced values of stress, and in the $\mathrm{Bi}_{2} \mathrm{Te}_{3}$ where they have increased in average in a homogenization process. This further explains what was already observed in Fig. 4, as in general in the copper the stresses in steady-state will be reduced and the opposite for the thermoelectric material.

It is interesting to note (as shown in Fig. 6 bottom) that the temperature evolution is not very affected by the presence of plasticity unlike the stress distribution. The latter is due to rather small contribution of the plastic dissipation towards total power sources, especially compared against the Joule dissipative term or Peltier cooling. 
Thus, it would appear that if the main goal in this problem concerned the temperature evolution, the plasticity would not have decisive role for computing the sufficiently good results.

\section{Conclusions}

A number of novelties for solving the coupled thermo-electro-mechanic problems for conductor materials are presented in this work. In particular, a complete formulation for thermo-electro-elastoplastic behavior has been developed in a consistent manner by using conservation principles along with the definition of a free-energy potential leading to corresponding constitutive relations. This formulation has been implemented in the most general 3D framework, by using 8-node hexahedral finite element for constructing semi-discretization, along with the global phase of time discretization by the Newmark scheme and local computation of the internal variables for plasticity. Finally, the thermo-electro-elastoplastic tangent modulus has been obtained assuming that every possible interaction is not negligible.

This development provides the sound basis to analyze practical examples, which either did not include an electric coupling to the thermomechanical formulation or have not implemented a plastic model in a thermo-electro-elastic framework. Moreover, the proposed formulation provides the starting point for further developments in plasticity that would include non-negligible magnetic field contribution.

The first validation numerical example has proven that all the couplings considered in this paper for the resulting element provide logica implemented, leading to the result that was expected, when the plastic behavior is not activated. In the second example, major differences between the elastic and plastic types of behavior have been found. In particular, the distribution of the stresses changes as the plasticity reduces the maximum value of von Mises by overcharging other parts of the domain, so the materials are more compensated. The increase of the plastic variables in the pulsed part affects the stress distribution in the steady-state, by reducing the maximum von Mises in the copper and the alumina and increasing it in the $\mathrm{Bi}_{2} \mathrm{Te}_{3}$ so a more complete study on the application of another pulses after irreversible changes take place is needed, and possibly a fatigue work to study the lifetime of the pulsed Peltier Cells. Also, the plastic dissipation in this example is not very significant when only the electric and the thermal fields are considered as the temperature in the cold side remains practically the same.

\section{Acknowledgments}

This work was supported jointly by Haut-de-France Region (CR Picardie) (120-2015-RDISTRUCT-000010 and RDISTRUCT-000010) and EU funding (FEDER) for Chaire-de-Mécanique-Numérique (contract Ref. 120-2015RDISTRUCTF-000010 and RDISTRUCTI-000004). This support is gratefully acknowledged.

\section{References}

[1] A. Ibrahimbegovic, Nonlinear Solid Mechanics: Theoretical Formulations and Finite Element Solution Methods, Vol. 160, Springer Science \& Business Media, 2009

[2] J.L. Ericksen, Introduction to the Thermodynamics of Solids, Springer, 1998

[3] G.A. Maugin, A.C. Eringen, Electrodynamics of Continua, Springer-Verlag, 1990.

[4] H. Allik, T.J.R. Hughes, Finite element method for piezoelectric vibration, Internat. J. Numer. Methods Engrg. 2 (2) (1970) $151-157$.

[5] P. Alotto, F. Freschi, M. Repetto, C. Rosso, The Cell Method for Electrical Engineering and Multiphysics Problems: An Introduction, Vol. 230, Springer Science \& Business Media, 2013.

[6] C.A. Balanis, Advanced Engineering Electromagnetics, ed: John Wiley \& Sons, 1989.

[7] J.D. Jackson, Classical Electrodynamics, Wiley, 1999.

[8] P. Bamberg, S. Sternberg, A Course in Mathematics for Students of Physics, Vol. 2, Cambridge University Press, 1991.

[9] P. Steinmann, D.K. Vu, Computational challenges in the simulation of nonlinear electroelasticity, Comput. Assist. Methods Eng. Sci. 19 (2012) 199-212.

[10] L.I. Anatychuk, O.J. Luste, Modern Thermodynamic Theory of Thermoelectricity. Thermoelectrics Handbook, CRC Press, 2006, (Chapter 2).

[11] J.E. Huber, N.A. Fleck, R.M. McMeeking, A crystal plasticity model for ferroelectrics, Ferroelectrics 228 (1) (1999) 39-52.

[12] C.M. Landis, Non-linear constitutive modeling of ferroelectrics, Curr. Opin. Solid State Mater. Sci. 8 (1) (2004) 59-69.

[13] R.C. Smith, A.G. Hatch, B. Mukherjee, S. Liu, A homogenized energy model for hysteresis in ferroelectric materials: General density formulation, J. Intell. Mater. Syst. Struct. 16 (9) (2005) 713-732.

[14] A. Sohrabi, A. Muliana, Rate-dependent electro-mechanical coupling response of ferroelectric materials: A finite element formulation, Mech. Mater. 62 (2013) 44-59. 
[15] J. Schröder, M. Labusch, M.A. Keip, Algorithmic two-scale transition for magneto-electro-mechanically coupled problems-FE2-scheme: Localization and homogenization, Comput. Methods Appl. Mech. Engrg. 302 (2016) 253-280.

[16] J.L. Pérez-Aparicio, R. Palma, R.L. Taylor, Finite element analysis and material sensitivity of Peltier thermoelectric cells coolers, Int. J. Heat Mass Transf. 55 (2012) 1363-1374.

[17] J.L. Pérez-Aparicio, R. Palma, R.L. Taylor, Multiphysics and thermodynamic formulations for equilibrium and non-equilibrium interactions: Non-linear finite elements applied to multi-coupled active materials, Arch. Comput. Methods Eng. 23 (3) (2016) 535-583.

[18] J.L. Pérez-Aparicio, R. Palma, P. Moreno-Navarro, Elasto-thermoelectric non-linear, fully coupled, and dynamic finite element analysis of pulsed thermoelectrics, Appl. Therm. Eng. 107 (2016) 398-409.

[19] A. Ibrahimbegovic, F. Gharzeddine, L. Chorfi, Classical plasticity and viscoplasticity models reformulated: theoretical basis and numerical implementation, Internat. J. Numer. Methods Engrg. 42 (8) (1998) 1499-1535.

[20] O.C. Zienkiewicz, R.L. Taylor, The Finite Element Method, Vols. I, II, III, Elsevier, 2005.

[21] T.J.R. Hughes, The Finite Element Method: Linear Static and Dynamic Finite Element Analysis, Prentice-Hall, Englewood Cliffs, NJ, 1987. 